\title{
Correlates and Determinants of Cardiorespiratory Fitness in Adults: a Systematic Review
}

\author{
Johannes Zeiher ${ }^{1 *}$ (D), Katherine J. Ombrellaro ${ }^{1}$, Nita Perumal², Thomas Keil ${ }^{3,4,5}$, Gert B. M. Mensink ${ }^{1}$ and
} Jonas D. Finger ${ }^{1}$

\begin{abstract}
Background: Enhanced cardiorespiratory fitness (CRF) is now a well-established predictor of numerous adverse health outcomes. Knowledge about the pathways leading to enhanced CRF is essential for developing appropriate interventions. Hence, the aim of this review was to provide a detailed overview of the current state of research regarding individual factors associated with or influencing CRF among the general adult population.

Methods: We searched the PubMed, EMBASE, and Cochrane Library databases and also conducted a search for grey literature (Google Scholar). Eligible indicators of CRF were objectively assessed measures of CRF by submaximal or maximal exercise testing measured using treadmill or cycle ergometer tests. We included quantitative observational studies of the general adult population. Using a semi-quantitative approach, we compiled summary tables aggregating the study results for each potential correlate or determinant of CRF.

Results: We identified 3005 studies, 78 of which met the inclusion criteria. Almost all of these studies were conducted in high-income countries. Study quality scores assessing the risk of bias in the individual studies ranged from 40 to 100\%. Male sex, age (inverse), education, socioeconomic status, ethnicity, body mass index (inverse), body weight (inverse), waist circumference, body fat (inverse), resting heart rate (inverse), C-reactive protein (inverse), smoking (inverse), alcohol consumption, and multiple measures of leisure-time physical activity were independently and consistently associated with CRF.

Conclusions: In synthesizing the current research on the correlates and determinants of CRF among adults, this systematic review identified gaps in the current understanding of factors influencing CRF. Beyond the scope of this review, environmental and interpersonal determinants should be further investigated.
\end{abstract}

Systematic Review Registration: PROSPERO, CRD42017055456.

Keywords: Cardiorespiratory fitness, Aerobic fitness, Risk factors, Individual factors, $\mathrm{VO}_{2 \mathrm{max}}$, Health behavior, Systematic review

\section{Key Points}

- This study is a systematic review of evidence concerning the correlates and determinants of CRF among adults in 78 included studies, which were conducted in 20 countries.

\footnotetext{
* Correspondence: zeiherj@rki.de

${ }^{1}$ Department of Epidemiology and Health Monitoring, Robert Koch Institute, General-Pape-Straße 62-66, 12101 Berlin, Germany

Full list of author information is available at the end of the article
}

- Whereas factors such as age and waist circumference were consistently associated with cardiorespiratory fitness, there was conflicting evidence for many other factors, revealing research gaps for future studies to address.

- This comprehensive summary of a large body of evidence may be used to develop evidence-based interventions to improve fitness levels in the general adult population. 


\section{Background}

A key aspect of the global strategy to tackle non-communicable diseases (NCDs) is the promotion of physical activity (PA) and the reduction of sedentary behavior $[79,80]$. PA has been linked to positive health outcomes, such as lower risks of ischemic heart disease, stroke, diabetes, and depression, and to a reduction in all-cause mortality [81]. Cardiorespiratory fitness (CRF) is another dimension of physical health linked to beneficial health outcomes. Whereas PA is behavioral and can be described as any bodily movement that is produced by skeletal muscles and requires energy exposure, CRF is a trait and is defined as the ability of the circulatory, respiratory, and muscular systems to supply oxygen during prolonged moderate-to-vigorous dynamic exercise [82, 83]. Therefore, PA and CRF are related, but not identical. CRF is usually measured using treadmill or ergometer exercise tests and is often expressed as maximal oxygen consumption $\left(\mathrm{VO}_{2 \max }\right)$, whereas PA is often assessed through self-report.

Over the past 30 years, research has shown that the positive effects of enhanced physical fitness, and especially CRF, are comparable to or even greater than those of PA [85-89]. In addition to predicting all-cause mortality, low CRF is an established predictor of cancer mortality [90], depression [91, 92], and metabolic syndrome $[93,94]$. Among the risk factors for cardiovascular disease (CVD), poor CRF has been found to be the most powerful predictor of morbidity [95].

Because of the importance of CRF in NCD prevention, it is crucial to better understand the correlates and determinants of CRF in the general population. CRF is known to be partly genetically determined [96, 97]. In addition to hereditary determinants and PA, many other individual and environmental factors are presumed to influence CRF [84, 88, 98]. A growing body of work links CRF to factors such as age [99], sex [100], smoking [8], alcohol consumption [101], body mass index (BMI) [23], educational status [102], and the residential built environment [103]. Although initial attempts have been made to develop a model of CRF and its determinants [84, 98], so far, there is no comprehensive model or framework that incorporates a wide range of influencing factors and the interrelations among them, as has been done in models of obesity [104, 105] and PA [106, 107]. Moreover, although researchers have systematically reviewed the factors associated with PA [106], to our knowledge, there has been no systematic review of the factors associated with CRF. Knowledge about the various pathways leading to the development of fitness is essential for creating appropriate interventions.

Hence, the aim of this systematic review was to provide a detailed overview of the current state of research regarding factors associated with ("correlates") or influencing ("determinants") CRF among the general adult population. Furthermore, we aimed to analyze the consistency of the reported associations. To narrow the study focus, we concentrated on individual factors associated with CRF, omitting interpersonal and environmental correlates and determinants.

\section{Methods}

\section{Protocol and Registration}

The review methodology, including the search strategy, data collection, and quality assessment of the included studies, was pre-specified and has been published in a review protocol [108]. The review was registered in the International Prospective Register of Systematic Reviews (PROSPERO, CRD42017055456). We followed the Preferred Reporting Items for Systematic Reviews and Metaanalyses (PRISMA) guidelines whenever applicable.

\section{Search Strategy and Eligibility Criteria}

Relevant studies were located from different sources: We searched the PubMed, EMBASE, and Cochrane Library databases from inception to Present. The last search was run on 1 February 2017. In addition, we conducted a search for grey literature (Google Scholar). A limited update literature search was performed from 01 February 2017 to 6 June 2019 on PubMed. We used a broad range of search terms for CRF measures (outcome; e.g., "cardiorespiratory fitness") and general correlates and determinants (exposures; e.g., "health behavior") to ensure that all potentially relevant articles investigating the factors associated with CRF were included [108]. We also cross-checked the references of the articles selected for full-text screening to locate additional studies. No language, text availability, publication status, or date restrictions were imposed. Eligible indicators of CRF were objectively assessed measures of CRF by submaximal or maximal exercise testing. Therefore, both direct indicators measured via spiroergometry and indirect indicators calculated via metabolic equations of oxygen consumption were included. We only included studies that assessed CRF by treadmill or cycle ergometer. The preferred laboratory measure of CRF (the "gold standard") is maximal oxygen consumption $\left(\mathrm{VO}_{2 \max }\right)$, which is measured in milliliters per kilogram per minute $(\mathrm{ml} / \mathrm{kg} /$ min) during exercise and reflects a person's maximal ability in terms of oxygen uptake, use, and transport [109]. $\mathrm{VO}_{2 \max }$ is defined as the point when oxygen consumption reaches a plateau and cannot be increased with an increase in effort [83, 110]. However, in exercise testing, such a clear plateau often cannot be achieved, and, instead, the highest obtained $\mathrm{VO}_{2}$ value, regardless the subject's effort, $\left(\mathrm{VO}_{2 \text { peak }}\right)$ is used [110]. Because the distinction between these two measures was not always clear in the included studies [111], this review uses the term $\mathrm{VO}_{2 \max }$ for both $\mathrm{VO}_{2 \max }$ and $\mathrm{VO}_{2 \text { peak }}$. 
To structure our search strategy, we used a conceptual framework that locates CRF on the pathway to NCDs and defines and categorizes potential factors associated with CRF. Based on the method recommended by Victoria et al. [112], we proposed this framework by adopting elements of different ecological models [108] (Fig. 1).

As mentioned above, to limit the scope of this systematic review, we focused on individual correlates and determinants of CRF and excluded environmental (e.g., public green spaces) and interpersonal (e.g., social support) factors. The following categories of factors were considered: (1) sociodemographic factors (e.g., age, sex, and education); (2) anthropometric measures (e.g., BMI, weight, and waist circumference [WC]; (3) vital parameters (e.g., resting heart rate [HR] and blood pressure [BP]); (4) comorbidities and medications; (5) biomarkers (e.g., C-reactive protein [CRP]); (6) PA parameters (e.g., leisure-time PA [LTPA]); and (7) other health-related behaviors (e.g., smoking and nutrition). Genetic factors, such as specific genetic variants associated with CRF trainability [113], were not included in this review.

In this review, we included quantitative observational studies (cohort studies, case-control studies, and crosssectional studies) reporting individual correlates of CRF

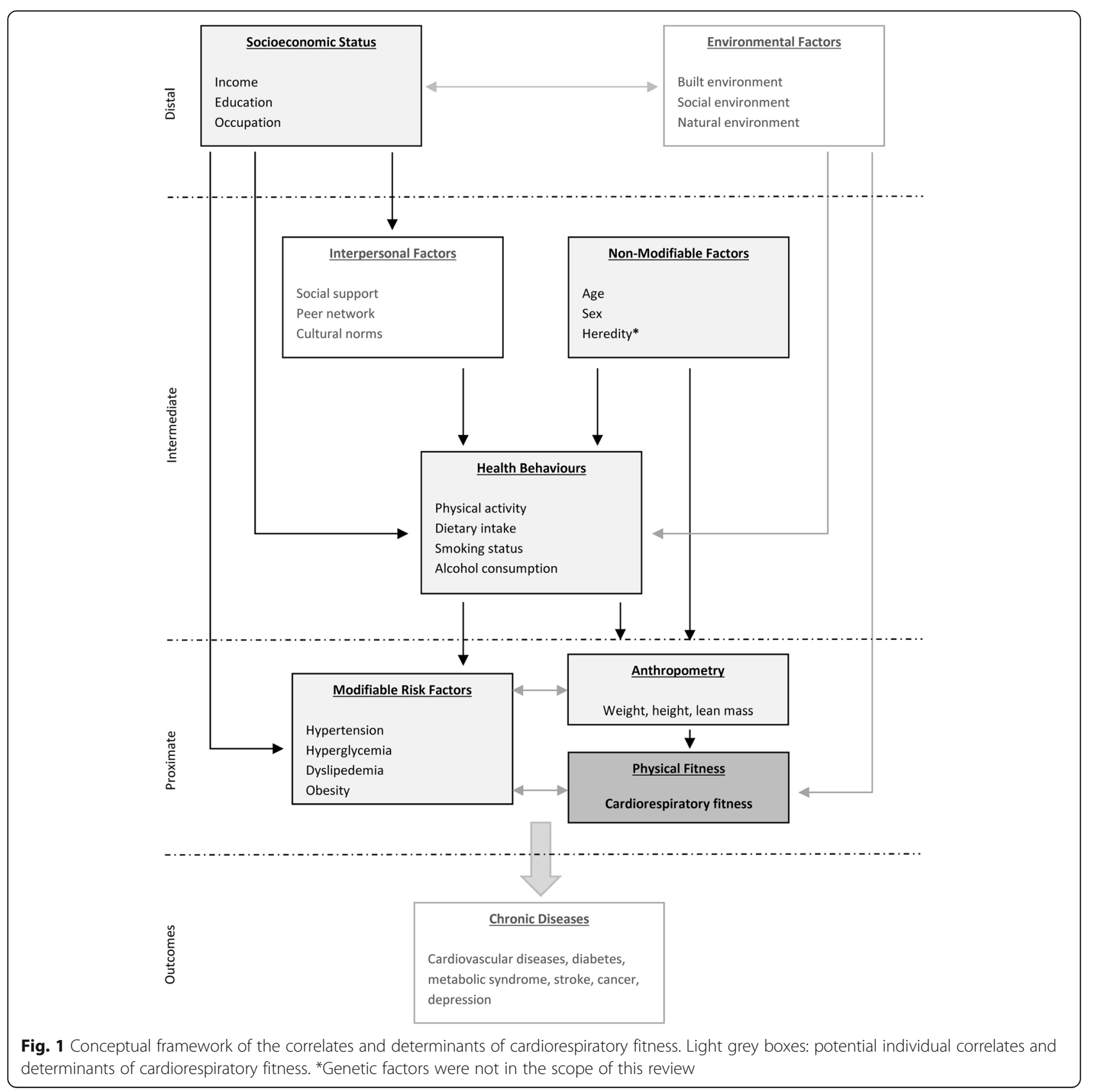


in the general adult population. The following exclusion criteria were applied: (1) studies on interventions or trials promoting CRF or PA; (2) studies focusing on children (aged 0-11 years), adolescents (aged 12-17 years), or older adults (aged 80+ years), although studies investigating a broad age range were not excluded and may include participants aged younger than 18 years or older than 80 years; (3) studies with highly select groups of participants not representative of CRF/PA in the general population (e.g., specific occupations such as firefighters or special forces, athletes, sports students, or institutionalized populations such as patients in hospitals or nursing homes); (4) studies presenting only univariate results that were unadjusted for confounding; (5) studies that assessed CRF by means other than bicycle ergometer or treadmill; and (6) reviews, letters to the editor, commentaries, and editorials.

\section{Data Extraction and Quality Assessment}

We imported all search results into Endnote X7 (Thomas Reuters, USA) reference management software and removed duplicates. At the first stage of screening, we reviewed the titles and abstracts. At the second stage, we assessed full-text articles for eligibility. The assessment was performed by two independent reviewers (NP and $\mathrm{KJO}$ at the first stage, JZ and $\mathrm{KJO}$ at the second stage). If the reviewers disagreed, a third reviewer was included in the discussion (JDF). Final decisions were made by consensus. From the final included studies, the following information was extracted and transferred to a detailed extraction worksheet, which was developed and pilot tested in advance: study characteristics (title, year, study name, country, and study period), study methods (e.g., study type, sample size, and inclusion/exclusion criteria), study population characteristics (e.g., age range and sex ratio), details of CRF assessment (e.g., test machine and exercise test protocol), analytical methods (e.g., statistical method and type of reported outcome), results, and reported limitations (Additional file 4: Data extraction table). When different multivariable models were reported for a sample, we extracted the fully adjusted models. The data extraction was conducted independently by two authors (JZ and KJO). Disagreements were solved by discussion; if agreement could not be reached, a third author (JDF) was involved, and decisions were made by a simple majority. The limited search update was performed by two authors (JZ and JDF [10\% random sample at title and abstract screening, full sample at full text screening]). Information from eligible studies retrieved in the update was extracted to a separate table (Additional file 3: Table OR1).

Additional information was obtained through a request to the author for one study, where further results were mentioned but not presented in detail [49]. The author responded, but this additional information was not used because the results violated the inclusion criteria. We contacted authors of two studies to clarify the direction of a reported association [18] and for further details about the study period [65]. Both of these authors responded, and the additional information was included in this study.

Risk of bias in each study was also assessed independently by two authors (JZ and KJO) using a customized version of the Quality Assessment Tool for Observational Cohort and Cross-Sectional Studies, published by the National Heart, Lung, and Blood Institute at the National Institutes of Health, USA [114]. We categorized risk of bias as "high" when a study reached $<50 \%$ of the fulfillment score, "moderate" when a study reached 50 $75 \%$, and "low" when $>75 \%$ of the criteria were fulfilled. Further details about this risk assessment procedure have been published elsewhere [108].

\section{Coding and Summarizing}

We compiled summary tables aggregating the existing research on each potential correlate or determinant of CRF. For this purpose, we modified the "semi-quantitative approach" originally proposed by Sallis et al. [115] (adapted by [116, 117]). This approach allowed summary measures to be calculated for each analyzed exposure, even when the heterogeneity among studies was high and no meta-analysis was possible. Following this approach, in the present article, we distinguish between the terms "study" and "sample." Each article included in this review is referred to as a study. Studies where the results were presented separately for men and women were counted as two samples. Studies that presented results only for the total population (men and women) and those that focused on only men or women were counted as one sample. Hereafter, we use the term "study" to refer to each article included in this review and the term "sample" to refer to each (sex-specific) sample or subsample.

For each sample, a significant direct or inverse association between CRF and the exposure is presented as "+" or "-" in the column "related to CRF"; non-significant associations are shown in the column "unrelated to CRF" ("O"). The findings are summarized by presenting the total number of samples and the numbers of samples with direct, indirect, and non-significant associations. Finally, following an approach applied in other semi-quantitative reviews [115-117], a summary measure for each association was calculated as follows: Agreement in direction in at least $60 \%$ of all samples was graded as a positive (“+”), negative (“-”), or non-significant association ("0"). If none of the categories had a majority of at least $60 \%$, the correlate was assessed as unclear ("?"). Outcomes that were heavily investigated (i.e., in at least 
ten independent samples) for which there was result agreement in at least $80 \%$ of the samples are denoted by "++," “- -," or "00." Summary measures were calculated only if an exposure was investigated in three or more individual samples; otherwise, the correlate was marked as not applicable ("n/a"). Each unique association is reported separately. Separate studies drawing on the same study population and reporting redundant exposures are presented in parentheses and were counted as one unit of analysis. For example, the two studies using the National Health and Nutrition Examination Survey data from the same study period that presented data on the association of age with CRF are both listed in the summary table but were counted as only one sample. In rare cases when separate studies using the same sample had contradictory results, we present the relevant details in a footnote.

\section{Outcome Variable: $C R F$}

For each sample included in this review, we extracted only one CRF measure, even if multiple measures were reported. Where it was assessed, we used the results for $\mathrm{VO}_{2 \max }$ because this is the gold standard for measuring CRF. If $\mathrm{VO}_{2 \max }$ was not reported, we summarize the results for the reported measures if they showed the same direction. When the results varied regarding the reported association with a specific exposure, we report the divergent results in a note at the end of the summary table. Additional information is also provided as a footnote to this table for cases where the association between CRF and a given exposure was non-linear (e.g., Ushaped) or the association was reported only for subgroups divided by variables other than sex (e.g., by ethnicity).

\section{Exposure Variables: Individual Correlates and Determinants of CRF}

We extracted each individual correlate or determinant of CRF and grouped them into the following categories: sociodemographic factors, anthropometric measures, vital parameters, comorbidities and medications, biomarkers, activity parameters, and other health behaviors. Correlates or determinants considered as individual factors that were not assignable to one of these categories (e.g., preterm birth) were grouped under "other." Where possible, we clustered similar factors to enable the calculation of summary measures.

We performed sensitivity analyses for all exposures that were reported in at least ten samples. We cross-tabulated the numbers of samples with positive associations, negative associations, and null findings by sex (men/ women/mixed sample), test machine (cycle ergometer/ treadmill), and CRF measure $\left(\mathrm{VO}_{2 \max }\right.$, direct $/ \mathrm{VO}_{2 \max }$, indirect/other) and checked for significant differences using Fisher's exact test. Using the same approach, we checked whether the results for BMI varied across CRF measures adjusting for body weight vs. measures that did not adjust for body weight.

\section{Results \\ Study Characteristics}

We identified a total of 78 articles for inclusion in this review. The complete selection process is shown in the PRISMA Flow Diagram (Fig. 2).

The initial search in PubMed, EMBASE, and the Cochrane Library yielded 3016 records. Following the removal of duplicates, 216 articles were added from Google Scholar and the reference cross-check. After title and abstract screening, 338 records remained for fulltext screening. A total of 260 of these records did not fulfil the eligibility criteria. All included studies were published in English. The dates of publication ranged from 1966 to 2017; the vast majority of articles were published after 2000 (see Fig. 3).

General characteristics of the studies reviewed can be found in Table 1.

Most studies were from North America (31) or Europe (29), and there were 14 from Asia and four from Oceania (including Australia). Thus, almost all studies were from high-income countries (74), according to the World Bank classification; only four were from lowermiddle or upper-middle-income countries, and none were from low-income countries. Except for one casecontrol study, all of the studies were cross-sectional (59) or cohort (18) studies. Almost two-thirds of the studies investigated both men and women (48), whereas eight studies reported results only for women and 17 reported results only for men. Five studies did not report the sex ratio of the study participants. The age range across the studies was 13 to 96 years. Sample sizes ranged from 79 to 218,820 . A variety of statistical techniques were used to investigate the associations between CRF and potential correlates and determinants, including general linear models such as analysis of variance; analysis of covariance; (multiple) linear, logistic, and quadratic regression; partial correlation; and general estimating equations.

The quality scores assessing the risk of bias in the individual studies ranged from 40 to $100 \%$ (Additional file 1: Table OR1.1). Thirty studies were classified as having low risk of bias, 41 were classified as having medium risk of bias, and seven were classified as having high risk of bias.

\section{CRF}

Half of the studies (39) used directly assessed $\mathrm{VO}_{2 \max }$ via gas analysis as the outcome variable, 19 reported estimated $\mathrm{VO}_{2 \max }$, and 19 used only measures other than $\mathrm{VO}_{2 \max }$ as the outcome variables. Directly assessed $\mathrm{VO}_{2 \max }$ via gas analysis was more common in studies with smaller sample sizes. No studies with more than 

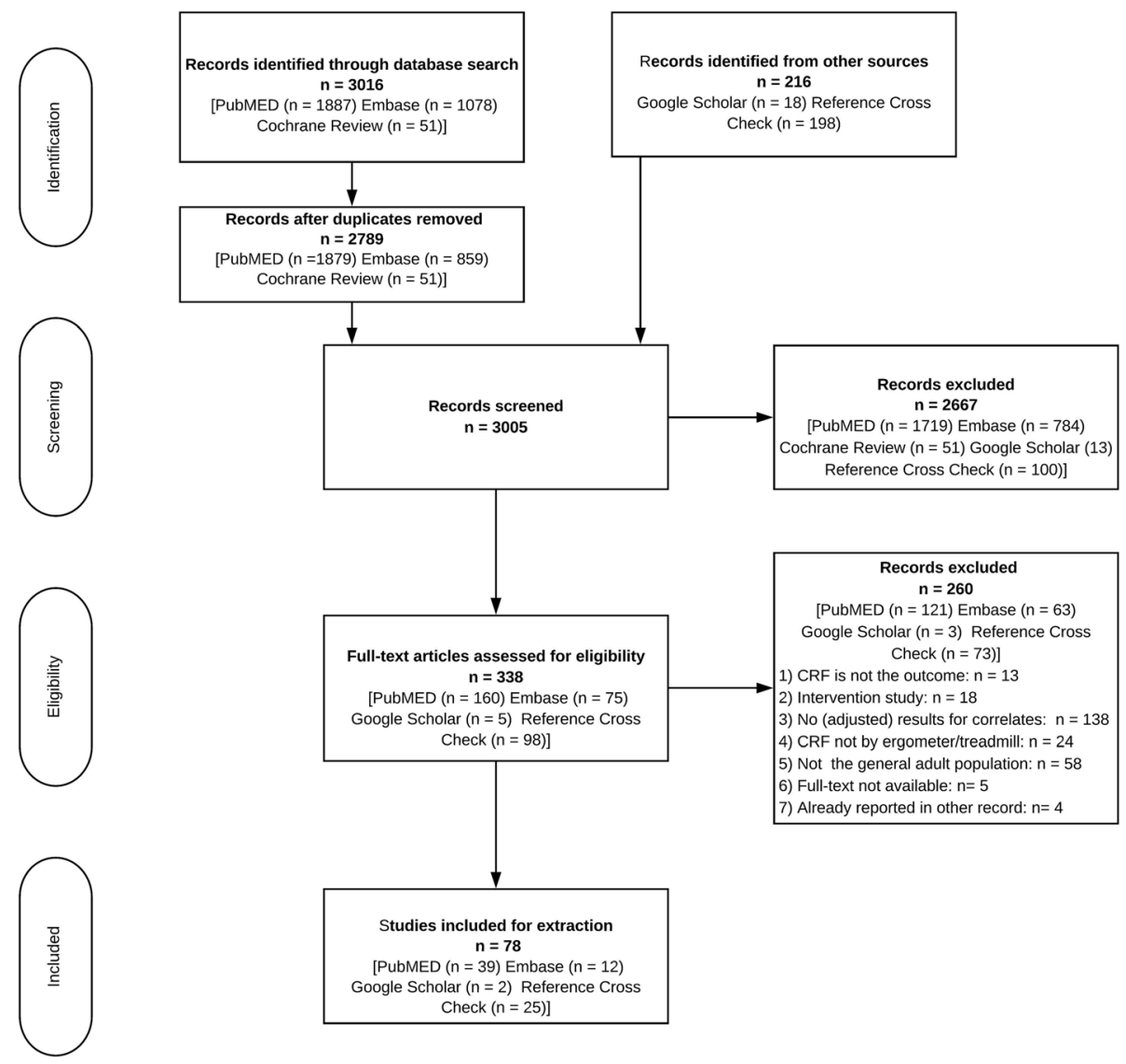

Fig. 2 PRISMA flow diagram. PRISMA: Preferred Reporting Items for Systematic Reviews and Meta-Analyses

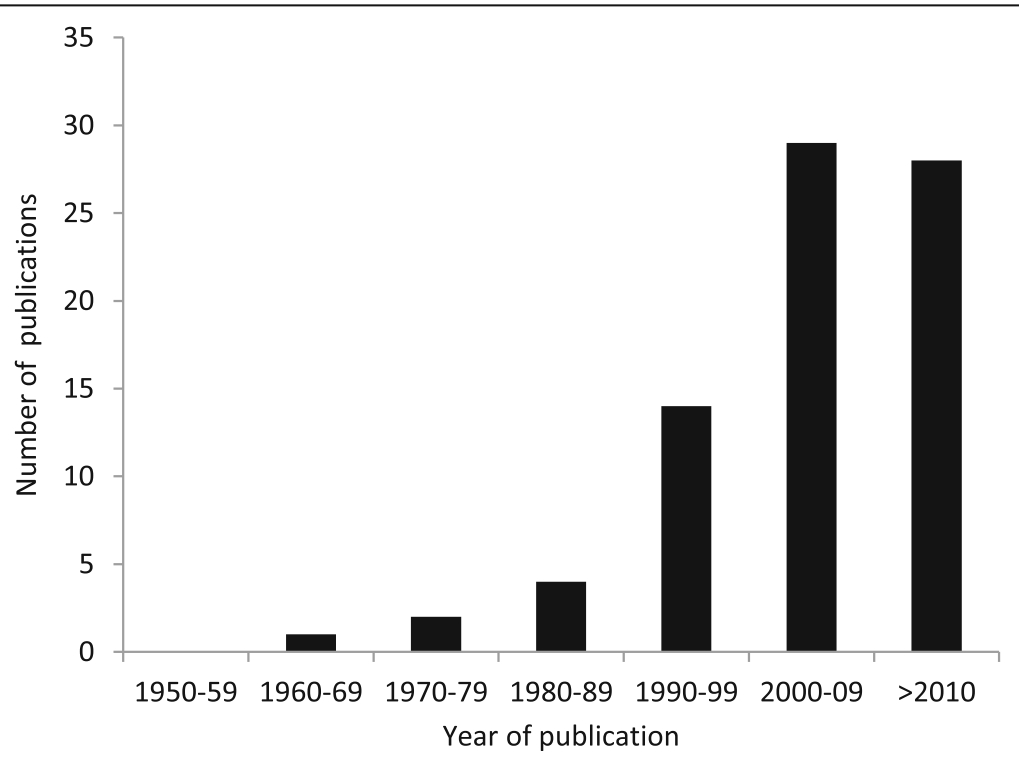

Fig. 3 Distribution of publications by year of publication $(n=78)$ 
Table 1 Included studies categorized by study characteristics

\begin{tabular}{|c|c|c|c|}
\hline & $\begin{array}{l}\text { Number of } \\
\text { studies }\end{array}$ & $\%$ & Reference numbers \\
\hline \multicolumn{4}{|l|}{ Risk of bias } \\
\hline Low & 30 & $38 \%$ & {$[1,2,4,12,14-16,18,20,23-26,31,34,36,40,42-44,49,50,57,59,60,62,67,75-77]$} \\
\hline Medium & 41 & $53 \%$ & {$[5-9,13,17,21,22,28-30,32,33,35,37-39,45-48,51,55,56,58,61,63-66,68-74,78]$} \\
\hline High & 7 & $9 \%$ & {$[3,19,27,41,52-54]$} \\
\hline \multicolumn{4}{|l|}{ Sample size } \\
\hline$<100$ & 5 & $6 \%$ & {$[5,17,41,53,71]$} \\
\hline 100-299 & 19 & $24 \%$ & {$[4,6,7,9,10,13,21,27,29,32,48,51,52,66,68,70,73,75,76]$} \\
\hline $300-499$ & 9 & $12 \%$ & {$[3,8,16,33,38,39,49,72,78]$} \\
\hline 500-1999 & 25 & $32 \%$ & {$[15,18,19,22-26,30,31,35,40,43,44,46,47,50,54,55,60,61,63,65,69,74]$} \\
\hline 2000-4999 & 14 & $18 \%$ & {$[1,12,14,20,34,36,42,45,56,57,59,62,67,77]$} \\
\hline$>5000$ & 6 & $8 \%$ & {$[2,11,28,37,58,64]$} \\
\hline \multicolumn{4}{|l|}{ Region } \\
\hline North America & 31 & $40 \%$ & {$[1,2,4,5,10-12,14,17,19,21,22,28,33,34,37,42-44,50-53,57,59,60,63,65,67,69,77]$} \\
\hline Europe & 29 & $37 \%$ & {$[6,7,18,20,23-27,29,30,36,38,40,45,47-49,55,56,62,64,66,70,71,75,76]$} \\
\hline Asia & 14 & $18 \%$ & {$[13,16,32,35,39,41,54,58,61,68,72-74,78]$} \\
\hline Oceania (including Australia) & 4 & $5 \%$ & {$[3,15,31,46]$} \\
\hline \multicolumn{4}{|c|}{ World Bank income classification } \\
\hline High-income countries & 74 & $95 \%$ & {$[1-29,33-60,62-71,74-78]$} \\
\hline $\begin{array}{l}\text { Upper-middle-income } \\
\text { countries }\end{array}$ & 2 & $3 \%$ & {$[72,73]$} \\
\hline $\begin{array}{l}\text { Lower-middle-income } \\
\text { countries }\end{array}$ & 2 & $3 \%$ & {$[32,61]$} \\
\hline Low-income countries & 0 & $0 \%$ & - \\
\hline \multicolumn{4}{|l|}{ Country } \\
\hline Canada & 1 & $1 \%$ & [51] \\
\hline United States & 30 & $38 \%$ & {$[1,2,4,5,10-12,14,17,19,21,22,28,33,34,37,42-44,50,52,53,57,59,60,63,65,67,69,77]$} \\
\hline Finland & 5 & $6 \%$ & {$[23,30,45,47,66]$} \\
\hline Sweden & 3 & $4 \%$ & {$[6,7,64]$} \\
\hline Norway & 3 & $4 \%$ & {$[18,55,62]$} \\
\hline Netherlands & 4 & $5 \%$ & {$[8,38,70,71]$} \\
\hline Germany & 6 & $8 \%$ & {$[20,25,26,29,36,40]$} \\
\hline United Kingdom & 2 & $3 \%$ & {$[24,49]$} \\
\hline Belgium & 2 & $3 \%$ & {$[48,76]$} \\
\hline Lithuania & 1 & $1 \%$ & {$[27]$} \\
\hline Italy & 1 & $1 \%$ & {$[56]$} \\
\hline Spain & 1 & $1 \%$ & {$[75]$} \\
\hline Israel & 3 & $4 \%$ & {$[16,35,58]$} \\
\hline Jordan & 1 & $1 \%$ & {$[32]$} \\
\hline India & 1 & $1 \%$ & [61] \\
\hline China & 2 & $3 \%$ & {$[72,73]$} \\
\hline Korea & 2 & $3 \%$ & {$[39,78]$} \\
\hline Japan & 5 & $6 \%$ & {$[13,41,54,68,74]$} \\
\hline Australia & 2 & $3 \%$ & {$[15]$} \\
\hline New Zealand & 2 & $3 \%$ & {$[31,46]$} \\
\hline
\end{tabular}


Table 1 Included studies categorized by study characteristics (Continued)

\begin{tabular}{|c|c|c|c|}
\hline & $\begin{array}{l}\text { Number of } \\
\text { studies }\end{array}$ & $\%$ & Reference numbers \\
\hline Multiple countries & 1 & $1 \%$ & [9] \\
\hline \multicolumn{4}{|l|}{ Study design } \\
\hline Cross-sectional & 59 & $76 \%$ & {$[1-3,9-14,16,18-21,23-30,32-36,39-45,47-52,54,56-59,61,62,65-69,72,74,75,77,78]$} \\
\hline Longitudinal & 18 & $23 \%$ & {$[6,15,17,22,31,37,38,46,53,55,60,63,64,70,73,76]$} \\
\hline Case-control & 1 & $1 \%$ & [71] \\
\hline \multicolumn{4}{|l|}{ Sex } \\
\hline Women only & 8 & $10 \%$ & {$[13,19,28,39,52,53,63,73]$} \\
\hline Men only & 17 & $22 \%$ & {$[3,5,10,17,23,32,35,45,47,48,50,55,56,61,64,74,76]$} \\
\hline Women and men & 48 & $62 \%$ & $\begin{array}{l}{[2,4,6-8,11,12,14-16,18,20-22,24-27,29,30,33,34,36-38,40-44,49,51,54,57-60,65-72,} \\
75,78,79]\end{array}$ \\
\hline NR & 5 & $6 \%$ & {$[1,9,31,46,62]$} \\
\hline \multicolumn{4}{|c|}{ Maximal or submaximal exercise testing } \\
\hline Maximal & 48 & $62 \%$ & $\begin{array}{l}{[8,11-13,16-18,21-23,25-30,33-40,45,47,48,51-53,55-57,59-61,63-66,69-71,73-75,} \\
77]\end{array}$ \\
\hline Submaximal & 27 & $35 \%$ & {$[1,5-7,9,14,15,19,20,24,31,41-44,46,49,50,54,58,62,67,68,72,76]$} \\
\hline NR & 3 & $4 \%$ & {$[10,32,78]$} \\
\hline \multicolumn{4}{|l|}{ Exercise test machine } \\
\hline Cycle & 34 & $44 \%$ & {$[3,5-7,9,13,15,20,23-27,30-32,36,40,45-48,51,55,56,64,66,68,71-76]$} \\
\hline Treadmill & 44 & $56 \%$ & $\begin{array}{l}{[1,2,4,8,10-12,14,16-19,21,22,28,29,33-35,37-39,41-44,49,50,52-54,57-63,65,67,69,} \\
70,77,78]\end{array}$ \\
\hline \multicolumn{4}{|l|}{ CRF measure } \\
\hline $\begin{array}{l}\mathrm{VO}_{2 \max } \text { direct (among } \\
\text { others) }\end{array}$ & 39 & $50 \%$ & {$[4,5,8,10,13,17,18,21-27,29,30,33,35,38-40,45,47-49,51-54,62,65,66,69-73,75,76]$} \\
\hline $\begin{array}{l}\mathrm{VO}_{2 \max } \text { indirect (among } \\
\text { others) }\end{array}$ & 19 & $24 \%$ & {$[1,6,7,9,14,16,31,41-44,46,50,67,68,74,77]$} \\
\hline Only other measures & 19 & $24 \%$ & {$[11,12,15,19,20,28,32,34,36,37,55-61,63,64]$} \\
\hline NR & 1 & $1 \%$ & [78] \\
\hline
\end{tabular}

5000 participants performed gas analysis to asses $\mathrm{VO}_{2 \max }$ (see Fig. 4).

In addition to $\mathrm{VO}_{2 \max }$, the following indicators of CRF were included in the selected studies: maximal physical working capacity (PWC) in Watts, PWC in Watts at variable and fixed HR thresholds (e.g., PWC at $75 \%$ of the predicted maximal heart rate or PWC at a heart rate of 170 beats per minute [PWC170]), time in seconds to HR threshold (e.g., exercise test duration to reach an $\mathrm{HR}$ of 130 beats per minute), energy expenditure in metabolic equivalents, and total exercise duration (in minutes or seconds). Eleven studies reported results for multiple measures of CRF. Almost two-thirds (48) of the studies applied maximal exercise testing (symptom limited), and one-third (27) applied submaximal testing. The treadmill (44) and the cycle ergometer (34) were commonly used exercise test machines in the included studies. Whereas the majority of European studies utilized cycle ergometers (76\%), almost all studies conducted in North America used treadmills for exercise testing (94\%; see Fig. 5).

\section{Exposure \\ Sociodemographic Characteristics}

Table 2 summarizes the adjusted associations between CRF and potential correlates or determinants. Unsurprisingly, the most studied individual correlate of CRF was age. More than $80 \%$ of the samples found a negative association with CRF, indicating that fitness declined with age. Three samples found no significant relation between age and CRF. A positive association was reported for two samples, but the age ranges were relatively narrow in these studies (21 to 43 years [12] and 18 to 30 years [12, 59]). 


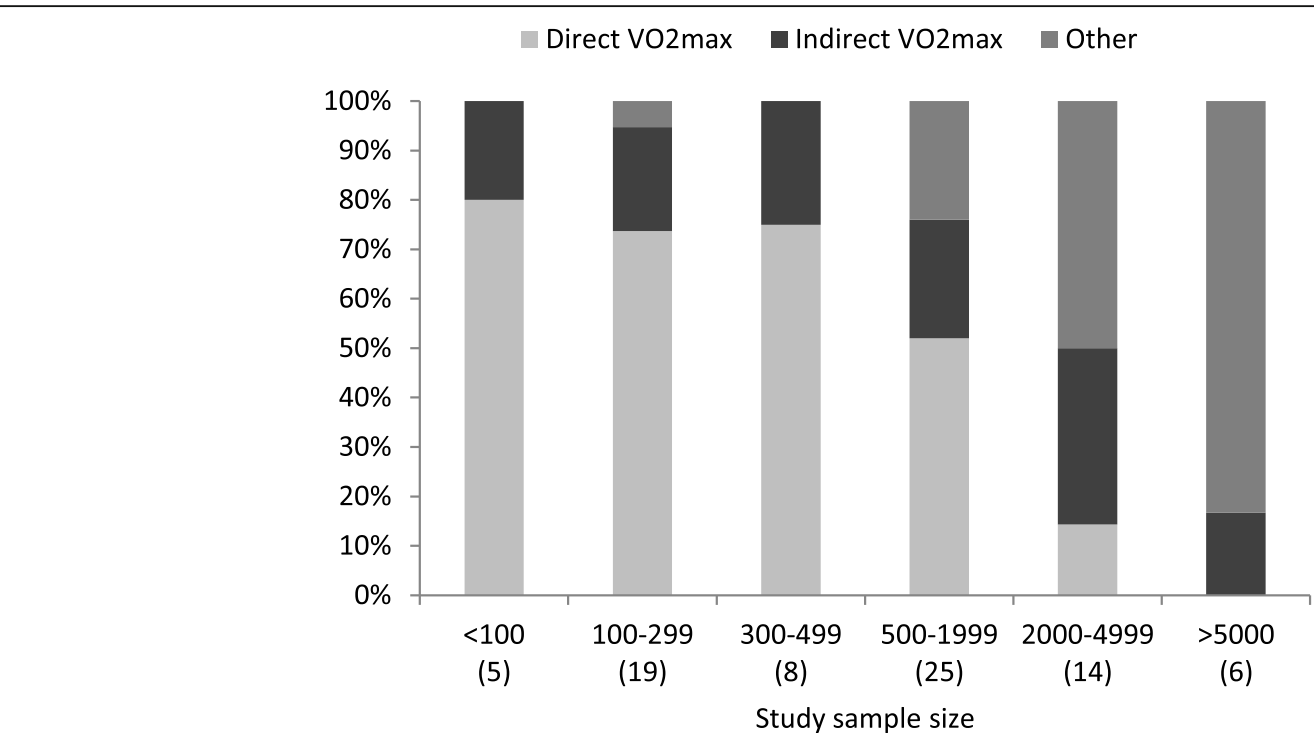

Fig. 4 Cardiorespiratory fitness measurement used by study sample size (the number of studies appears in parentheses. The studies sum to $n=77$ because one study did not report whether they used direct or indirect $\left.V^{2 \text { max }}\right) V O_{2 \max }$ maximal oxygen consumption

Sex differences were investigated in six studies, all of which reported significantly higher CRF among men than among women.

The association between CRF and ethnicity was investigated in five samples, all in the USA. In four samples, higher CRF was observed among "whites" than among "blacks," whereas one sample of men observed no association between ethnicity and CRF.

We identified 19 samples investigating the associations between CRF measures and socioeconomic status (SES) variables. The most common SES measure was education (ten samples), and a positive association between education and CRF was reported for a majority of the samples, although in some samples no significant association was observed. Composite SES indexes were investigated in three samples, with summary measures also showing a positive association with CRF.

\section{Anthropometric Measures}

Anthropometric measures were frequently investigated in the included studies. BMI, the most frequently analyzed anthropometric factor (24 samples), showed an inverse association with CRF, as did WC and body fat. Weight and total lean mass were positively associated with CRF in at least $60 \%$ of the samples.

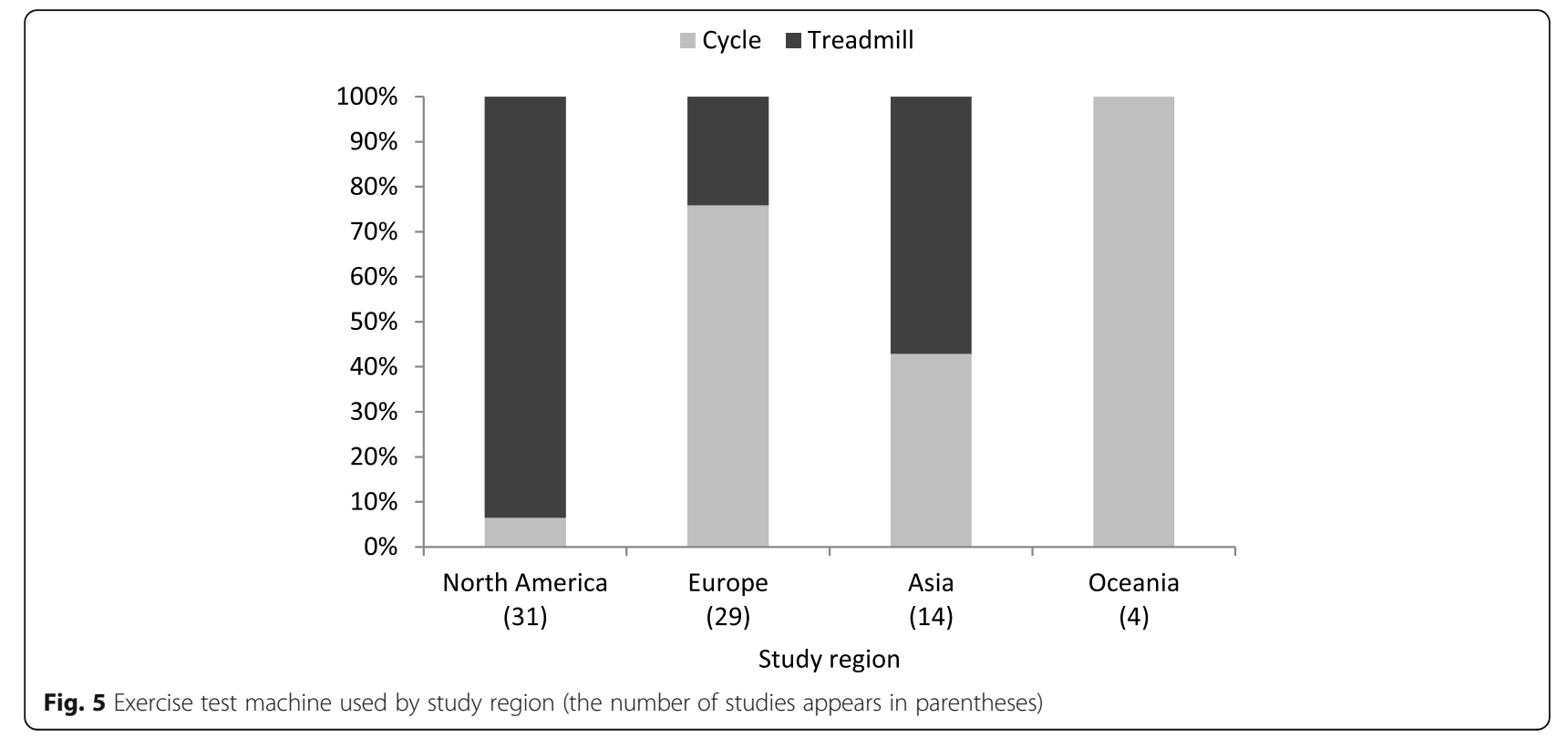


Table 2 Summary of individual correlates and determinants of cardiorespiratory fitness

\begin{tabular}{|c|c|c|c|c|c|c|c|c|}
\hline & \multicolumn{2}{|l|}{ Related to CRF } & \multirow{2}{*}{$\begin{array}{l}\text { Unrelated to CRF } \\
\text { Reference numbers }\end{array}$} & \multicolumn{5}{|c|}{ Summary of associations } \\
\hline & Reference numbers & $\begin{array}{l}\text { Association } \\
(+/-)\end{array}$ & & $\#$ & $\#+$ & $\#-$ & $\# 0$ & $\begin{array}{l}\text { Association } \\
(+/-)\end{array}$ \\
\hline \multicolumn{9}{|c|}{ Sociodemographic characteristics } \\
\hline \multirow[t]{2}{*}{ Age } & $23,\left(12 m^{36}, 59 m^{36}\right)$ & + & \multirow{2}{*}{$\begin{array}{l}\left(12 f^{15}, 59 f^{17}\right), 60 f \\
68 m\end{array}$} & \multirow[t]{2}{*}{43} & \multirow[t]{2}{*}{2} & \multirow[t]{2}{*}{38} & \multirow[t]{2}{*}{3} & \multirow[t]{2}{*}{--} \\
\hline & $\begin{array}{l}3 \mathrm{~m}, 5 \mathrm{~m}, 13 \mathrm{f},(14,67), 16 \mathrm{f}, 16 \mathrm{~m}, 17 \mathrm{~m}, 18,22,24,27 \mathrm{f} \\
27 \mathrm{~m}, 28 \mathrm{f}, 29 \mathrm{f}, 29 \mathrm{~m}, 30 \mathrm{~m}, 32 \mathrm{~m}, 33,35 \mathrm{~m}, 37 \mathrm{f}, 37 \mathrm{~m}, 39 \mathrm{f} \\
40,45 \mathrm{~m}, 47 \mathrm{~m}, 53 \mathrm{f}, 54 \mathrm{f}, 54 \mathrm{~m}, 56 \mathrm{~m}^{16}, 60 \mathrm{~m}^{18}, 61 \mathrm{~m}, 65 \mathrm{f} \\
65 \mathrm{~m}, 68 \mathrm{f}, 69 \mathrm{f}, 69 \mathrm{~m}, 72 \mathrm{f}, 72 \mathrm{~m}\end{array}$ & - & & & & & & \\
\hline Sex (women vs. men) & $(14,67), 22,24,33,40,18$ & - & & 6 & & 6 & & - \\
\hline Ethnicity & $19 f, 12 f, 12 m^{37}, 14 f$ & & $14 m$ & 5 & & & & $\mathrm{n} / \mathrm{a}$ \\
\hline $\begin{array}{l}\text { Marital status (married } \\
\text { vs. single) }\end{array}$ & $45 m$ & + & & 1 & 1 & & & $\mathrm{n} / \mathrm{a}$ \\
\hline \multicolumn{9}{|l|}{ Socioeconomic status } \\
\hline Education & $6 f,\left(12 m^{5}, 59 m^{7}\right),\left(12 f^{5}, 59 f\right), 23 m, 45 m, 58^{6}$ & + & $\begin{array}{l}7 f, 7 m,(14 m, 67), \\
(14 f, 67)\end{array}$ & 10 & 6 & & 4 & + \\
\hline Parental education & $15^{38}$ & - & & 1 & & 1 & & $\mathrm{n} / \mathrm{a}$ \\
\hline Maternal education & $15^{39}$ & + & & 1 & 1 & & & $\mathrm{n} / \mathrm{a}$ \\
\hline Paternal education & $15^{40}$ & - & & 1 & & 1 & & $\mathrm{n} / \mathrm{a}$ \\
\hline Composite SES index & $20 f, 58$ & + & $20 \mathrm{~m}$ & 3 & 2 & & 1 & + \\
\hline Occupation & $58,45 m^{14}$ & + & & 2 & 2 & & & $\mathrm{n} / \mathrm{a}$ \\
\hline Employment & $45 m$ & + & & 1 & & & & $\mathrm{n} / \mathrm{a}$ \\
\hline Working mother & & & $6 \mathrm{~m}$ & 1 & & & 1 & $\mathrm{n} / \mathrm{a}$ \\
\hline Income & $45 m$ & + & & 1 & 1 & & & $\mathrm{n} / \mathrm{a}$ \\
\hline Financial strain & $58^{13}$ & + & & 1 & 1 & & & $\mathrm{n} / \mathrm{a}$ \\
\hline \multicolumn{9}{|l|}{ Anthropometric measures } \\
\hline \multirow[t]{2}{*}{ Body mass index } & $\begin{array}{l}10 \mathrm{~m}, 13 \mathrm{f}, 16 \mathrm{f}, 18,23 \mathrm{~m}, 37 \mathrm{~m}, 37 \mathrm{f}, 59 \mathrm{~m}, 59 \mathrm{f}, 64 \mathrm{~m}^{1}, 65 \mathrm{~m} \\
65 \mathrm{f}, 66 \mathrm{~m}, 66 \mathrm{f}, 77 \mathrm{~m}, 77 \mathrm{f}, 78 \mathrm{~m}, 78 \mathrm{f}\end{array}$ & - & $7 f, 7 m, 16 m$ & 24 & 3 & 18 & 3 & - \\
\hline & $32 m, 40,56 m$ & + & & & & & & \\
\hline Overweight & $63 f$ & - & 67 & 2 & & 1 & 1 & $\mathrm{n} / \mathrm{a}$ \\
\hline Obese & $63 f, 67$ & - & & 2 & & 2 & & $\mathrm{n} / \mathrm{a}$ \\
\hline Relative weight & $11 \mathrm{~m}, 11 \mathrm{f}$ & - & & 2 & & 2 & & $\mathrm{n} / \mathrm{a}$ \\
\hline Body shape & & & $3 m$ & 1 & & & 1 & $\mathrm{n} / \mathrm{a}$ \\
\hline Waist circumference & $13 f,(14 m, 77 m), 16 m, 16 f, 23 m, 30 m, 30 f, 78 m, 78 f$ & - & $(77 f, 14 f)^{8}$ & 10 & & 9 & 1 & -- \\
\hline $\begin{array}{l}\text { Waist-to-hip } \\
\text { circumference ratio }\end{array}$ & $47 m$ & - & & 1 & & 1 & & $\mathrm{n} / \mathrm{a}$ \\
\hline \multirow[t]{2}{*}{ Weight } & $6 \mathrm{~m}$ & - & & 4 & 3 & 1 & & + \\
\hline & $24,35 m, 47 m$ & + & & & & & & \\
\hline Body fat (\%) & $10 \mathrm{~m}, 33 \mathrm{~m}, 33 \mathrm{f}, 39 \mathrm{f}, 61 \mathrm{~m}, 68 \mathrm{~m}, 68 \mathrm{f}^{2}$ & - & & 7 & & 7 & & + \\
\hline Total fat mass & $72 f, 72 m$ & - & & 2 & & 2 & & $\mathrm{n} / \mathrm{a}$ \\
\hline Lean mass (\%) & 9,22 & + & & 2 & 2 & & & $\mathrm{n} / \mathrm{a}$ \\
\hline Total lean mass & $72 m, 72 f$ & + & 9 & 3 & 2 & & 1 & + \\
\hline Skeletal muscle mass & $54 m, 54 f$ & + & & 2 & 2 & & & $\mathrm{n} / \mathrm{a}$ \\
\hline Sum subscapular & $12 m, 12 f$ & - & & 2 & & 2 & & $\mathrm{n} / \mathrm{a}$ \\
\hline $\begin{array}{l}\text { Appendicular lean } \\
\text { mass/height squared }\end{array}$ & & & 9 & 1 & & & 1 & $\mathrm{n} / \mathrm{a}$ \\
\hline
\end{tabular}


Table 2 Summary of individual correlates and determinants of cardiorespiratory fitness (Continued)

\begin{tabular}{|c|c|c|c|c|c|c|c|c|}
\hline & \multicolumn{2}{|l|}{ Related to CRF } & \multirow{2}{*}{$\begin{array}{l}\text { Unrelated to CRF } \\
\text { Reference numbers }\end{array}$} & \multicolumn{5}{|c|}{ Summary of associations } \\
\hline & Reference numbers & $\begin{array}{l}\text { Association } \\
(+/-)\end{array}$ & & $\#$ & $\#+$ & $\#-$ & $\# 0$ & $\begin{array}{l}\text { Association } \\
(+/-)\end{array}$ \\
\hline $\begin{array}{l}\text { Appendicular lean mass } \\
(\%)\end{array}$ & 9 & + & & 1 & 1 & & & $\mathrm{n} / \mathrm{a}$ \\
\hline Height & $35 m$ & + & $10 \mathrm{~m}$ & 2 & 1 & & 1 & $\mathrm{n} / \mathrm{a}$ \\
\hline Birth weight & $64 m$ & + & & 1 & 1 & & & $\mathrm{n} / \mathrm{a}$ \\
\hline \multicolumn{9}{|l|}{ Vital parameters } \\
\hline Heart rate, resting & $10 \mathrm{~m}, 11 \mathrm{~m}, 11 \mathrm{f}, 47 \mathrm{~m}, 56 \mathrm{~m}, 67$ & - & & 6 & & 6 & & - \\
\hline $\begin{array}{l}\text { Heart rate, maximal } \\
\text { exercise }\end{array}$ & $47 m$ & + & & 1 & 1 & & & $n / a$ \\
\hline $\begin{array}{l}\text { Heart rate, after CRF } \\
\text { test }\end{array}$ & $66 m, 66 f^{4}$ & - & & 2 & & 2 & & $\mathrm{n} / \mathrm{a}$ \\
\hline Mean blood pressure & $56 \mathrm{~m}, 68$ & - & & 2 & & 2 & & $\mathrm{n} / \mathrm{a}$ \\
\hline Systolic blood pressure & $12 \mathrm{~m}, 12 \mathrm{f}, 77 \mathrm{~m}$ & - & $77 f$ & 4 & & 3 & 1 & - \\
\hline Diastolic blood pressure & $77 m, 77 f$ & - & & 2 & & 2 & & $n / a$ \\
\hline $\begin{array}{l}\text { Forced expiratory } \\
\text { volume in } 1 \mathrm{~s}\end{array}$ & $47 m, 59 m^{3}, 59 f^{3}$ & + & & 3 & 3 & & & + \\
\hline Vital capacity & $11 \mathrm{~m}, 11 \mathrm{f}, 51$ & + & & 3 & 3 & & & + \\
\hline $\begin{array}{l}\text { Aortic augmentation } \\
\text { index }\end{array}$ & $10 \mathrm{~m}$ & - & & 1 & & 1 & & $\mathrm{n} / \mathrm{a}$ \\
\hline \multicolumn{9}{|c|}{ Comorbidities and medications } \\
\hline Coronary heart disease & $47 m$ & - & & 1 & & 1 & & $\mathrm{n} / \mathrm{a}$ \\
\hline Asthma & $47 m$ & - & & 1 & & 1 & & $n / a$ \\
\hline Hypertension & & & 67 & 1 & & & 1 & $\mathrm{n} / \mathrm{a}$ \\
\hline Diabetes & & & 67 & 1 & & & 1 & n/a \\
\hline Beta-blocker use & $30 f, 30 m$ & - & & 2 & & 2 & & $n / a$ \\
\hline $\begin{array}{l}\text { Shortness of breath } \\
\text { upon exertion }\end{array}$ & & & $32 \mathrm{~m}$ & 1 & & & 1 & $n / a$ \\
\hline \multicolumn{9}{|l|}{ Biomarkers } \\
\hline Bicarbonate & 1 & + & & 1 & 1 & & & $\mathrm{n} / \mathrm{a}$ \\
\hline Anion gap & 1 & - & & 1 & & 1 & & n/a \\
\hline Vitamin D & 4 & + & & 1 & 1 & & & $\mathrm{n} / \mathrm{a}$ \\
\hline $\begin{array}{l}\text { (High-sensitivity) C- } \\
\text { reactive protein }\end{array}$ & $2,41,44 f, 44 m$ & - & 67 & 5 & & 4 & 1 & - \\
\hline $\begin{array}{l}\text { Thyroid-stimulating } \\
\text { hormone }\end{array}$ & $36^{9}$ & & & 1 & & & & $\mathrm{n} / \mathrm{a}$ \\
\hline Hemoglobin & $47 m$ & + & & 1 & 1 & & & $\mathrm{n} / \mathrm{a}$ \\
\hline Fasting serum insulin & $47 m$ & - & 24 & 2 & & 1 & 1 & $\mathrm{n} / \mathrm{a}$ \\
\hline $\begin{array}{l}\text { 2-h glucose tolerance } \\
\text { test }\end{array}$ & & & 24 & 1 & & & 1 & $\mathrm{n} / \mathrm{a}$ \\
\hline Glucose, mg/dL & & & $77 m, 77 f$ & 2 & & & 2 & $\mathrm{n} / \mathrm{a}$ \\
\hline $\mathrm{HbA} 1 \mathrm{c}, \%$ & & & $77 m, 77 f$ & 2 & & & 2 & $\mathrm{n} / \mathrm{a}$ \\
\hline Ferritin & $50 m^{10}$ & - & & 1 & & 1 & & $\mathrm{n} / \mathrm{a}$ \\
\hline $\begin{array}{l}\text { High-density } \\
\text { lipoprotein (HDL) } \\
\text { cholesterol }\end{array}$ & $56 m^{11}, 77 m, 77 f$ & + & & 3 & 3 & & & + \\
\hline
\end{tabular}


Table 2 Summary of individual correlates and determinants of cardiorespiratory fitness (Continued)

\begin{tabular}{|c|c|c|c|c|c|c|c|c|}
\hline & \multicolumn{2}{|l|}{ Related to CRF } & \multirow{2}{*}{$\begin{array}{l}\text { Unrelated to CRF } \\
\text { Reference numbers }\end{array}$} & \multicolumn{5}{|c|}{ Summary of associations } \\
\hline & Reference numbers & $\begin{array}{l}\text { Association } \\
(+/-)\end{array}$ & & $\#$ & $\#+$ & $\#-$ & $\# 0$ & $\begin{array}{l}\text { Association } \\
(+/-)\end{array}$ \\
\hline Non-HDL cholesterol & $56 m^{12}$ & + & & 1 & 1 & & & $n / a$ \\
\hline Cholesterol & $77 f$ & - & $(67,77 m)$ & 2 & & 1 & 1 & $n / a$ \\
\hline Triglycerides & $77 m^{42}$ & & $77 f$ & 2 & & & 1 & $\mathrm{n} / \mathrm{a}$ \\
\hline Creatinine excretion & $21 \mathrm{f}, 21 \mathrm{~m}$ & + & & 2 & 2 & & & $\mathrm{n} / \mathrm{a}$ \\
\hline White blood cell count & & & 67 & 1 & & & 1 & $\mathrm{n} / \mathrm{a}$ \\
\hline Homocysteine & $43 f$ & - & $43 m$ & 2 & & 1 & 1 & $\mathrm{n} / \mathrm{a}$ \\
\hline $\begin{array}{l}\text { Insulin-like growth } \\
\text { factor (IGF) I }\end{array}$ & & & $25 f, 25 m$ & 2 & & & 2 & $\mathrm{n} / \mathrm{a}$ \\
\hline $\begin{array}{l}\text { IGF-binding protein } 3 \\
\text { (IGFBP-3) }\end{array}$ & & & $25 f, 25 m$ & 2 & & & 2 & $\mathrm{n} / \mathrm{a}$ \\
\hline $\begin{array}{l}\text { IGF-I and IGF-I/IGFBP-3 } \\
\text { ratio }\end{array}$ & & & $25 f, 25 m$ & 2 & & & 2 & $n / a$ \\
\hline Nonesterified fatty acid & & & 24 & 1 & & & 1 & $\mathrm{n} / \mathrm{a}$ \\
\hline $\begin{array}{l}\text { Red cell distribution } \\
\text { width }\end{array}$ & 9 & - & & 1 & & 1 & & $\mathrm{n} / \mathrm{a}$ \\
\hline Flow-mediated dilation & & & 26 & 1 & & & 1 & $n / a$ \\
\hline $\begin{array}{l}\text { Nitroglycerin-mediated } \\
\text { dilation }\end{array}$ & $26^{33}$ & + & & 1 & 1 & & & $\mathrm{n} / \mathrm{a}$ \\
\hline \multicolumn{9}{|l|}{ Activity parameters } \\
\hline \multicolumn{9}{|l|}{ Subjective measurements } \\
\hline \multicolumn{9}{|l|}{ Overall PA } \\
\hline PA index, various & $\begin{array}{l}11 \mathrm{f}, 11 \mathrm{~m},(12 \mathrm{f}, 59 \mathrm{f}),(12 \mathrm{~m}, 59 \mathrm{~m}), 33 \mathrm{f}, 33 \mathrm{~m}, 37 \mathrm{f}, 37 \mathrm{~m}, 38 \\
48 \mathrm{~m}, 49 \mathrm{~m}, 56 \mathrm{~m}, 60 \mathrm{f}^{9}, 60 \mathrm{~m}^{29}\end{array}$ & + & $\begin{array}{l}10 m, 49 f, 65 f, 65 m \\
76 m\end{array}$ & 19 & 14 & & 5 & + \\
\hline PA level, high vs. low & $733^{2}$ & + & $49 f, 49 m$ & 3 & 1 & & 2 & \\
\hline $\begin{array}{l}\text { Failure to meet PA } \\
\text { recommendation }\end{array}$ & & & $63 f$ & 1 & & & 1 & $n / a$ \\
\hline PA times/week & $23 m, 47 m, 66 f, 66 m$ & + & & 4 & 4 & & & + \\
\hline $\begin{array}{l}\text { PA duration (hours/ } \\
\text { week) }\end{array}$ & $45 \mathrm{~m}$ & + & & 1 & 1 & & & $n / a$ \\
\hline $\begin{array}{l}\text { PA (in METS or MET } \\
\text { minutes/week) }\end{array}$ & $\left(14 f, 67^{30)},\left(14 m, 67^{30)}, 38,(45 m, 47 m), 68 m^{31}, 70\right.\right.$ & + & $68 f$ & 7 & 6 & & 1 & + \\
\hline $\begin{array}{l}\text { Energy expenditure } \\
\text { (kcal/week) }\end{array}$ & $45 \mathrm{~m}$ & + & & 1 & 1 & & & $n / a$ \\
\hline $\begin{array}{l}\text { Moderate-to-vigorous } \\
\text { PA }\end{array}$ & $30 f, 30 \mathrm{~m}$ & + & & 2 & 2 & & & $n / a$ \\
\hline Moderate PA & $39 f$ & + & 75 & 2 & 1 & & 1 & $\mathrm{n} / \mathrm{a}$ \\
\hline Vigorous PA & 75 & + & & 1 & 1 & & & $\mathrm{n} / \mathrm{a}$ \\
\hline $\begin{array}{l}\text { Proportion of } \\
\text { vigorous PA/all PA }\end{array}$ & $14 f, 14 m$ & + & & 2 & 2 & & & $n / a$ \\
\hline \multicolumn{9}{|l|}{ LTPA } \\
\hline Regular exercise & & & $32 m$ & 1 & & & 1 & $\mathrm{n} / \mathrm{a}$ \\
\hline Activity $>2 \mathrm{~h} /$ week & $35 \mathrm{~m}$ & + & & 1 & 1 & & & $\mathrm{n} / \mathrm{a}$ \\
\hline $\begin{array}{l}\text { Training time (hours/ } \\
\text { week) }\end{array}$ & $16 f, 16 \mathrm{~m}$ & - & & 2 & & 2 & & $\mathrm{n} / \mathrm{a}$ \\
\hline $\begin{array}{l}\text { Leisure sports } \\
\text { activities (yes vs. no) }\end{array}$ & $7 f, 74 m$ & + & & 2 & 2 & & & $\mathrm{n} / \mathrm{a}$ \\
\hline LTPA, quartiles & 22 & + & & 1 & 1 & & & $\mathrm{n} / \mathrm{a}$ \\
\hline
\end{tabular}


Table 2 Summary of individual correlates and determinants of cardiorespiratory fitness (Continued)

\begin{tabular}{|c|c|c|c|c|c|c|c|c|}
\hline & \multicolumn{2}{|l|}{ Related to CRF } & \multirow{2}{*}{$\begin{array}{l}\text { Unrelated to CRF } \\
\text { Reference numbers }\end{array}$} & \multicolumn{5}{|c|}{ Summary of associations } \\
\hline & Reference numbers & $\begin{array}{l}\text { Association } \\
(+/-)\end{array}$ & & \# & $\#+$ & \#- & $\# 0$ & $\begin{array}{l}\text { Association } \\
(+/-)\end{array}$ \\
\hline $\begin{array}{l}\text { Intensity of LTPA (in } \\
\text { METS) }\end{array}$ & $61 \mathrm{~m}$ & + & & 1 & 1 & & & $\mathrm{n} / \mathrm{a}$ \\
\hline LTPA, duration/day & & & $61 \mathrm{~m}$ & 1 & & & 1 & $\mathrm{n} / \mathrm{a}$ \\
\hline $\begin{array}{l}\text { Energy expenditure } \\
\text { during active leisure } \\
\text { time }\end{array}$ & 48 & + & & 1 & 1 & & & $\mathrm{n} / \mathrm{a}$ \\
\hline $\begin{array}{l}\text { Caloric expenditure } \\
\text { in sports activity }\end{array}$ & $16 f, 16 \mathrm{~m}$ & + & & 2 & 2 & & & $\mathrm{n} / \mathrm{a}$ \\
\hline $\begin{array}{l}\text { Membership in a } \\
\text { sports club }\end{array}$ & & & $7 m$ & 1 & & & 1 & $\mathrm{n} / \mathrm{a}$ \\
\hline $\begin{array}{l}\text { Past participation } \\
\text { (years of vigorous or } \\
\text { moderate sporting } \\
\text { activities) }\end{array}$ & & & $49 f, 49 m$ & 2 & & & 2 & $\mathrm{n} / \mathrm{a}$ \\
\hline \multicolumn{9}{|l|}{ Occupational PA } \\
\hline Occupational PA & $74 m$ & + & $45 m$ & 2 & 1 & & 1 & $n / a$ \\
\hline \multicolumn{9}{|l|}{ Sedentary PA } \\
\hline Sedentary PA & $39 f$ & - & & 1 & & 1 & & $n / a$ \\
\hline \multicolumn{9}{|l|}{ Other PA measures } \\
\hline $\begin{array}{l}\text { Satisfied with sports } \\
\text { performance (yes) }\end{array}$ & & & $7 f, 7 m$ & 2 & & & 2 & $\mathrm{n} / \mathrm{a}$ \\
\hline $\begin{array}{l}\text { Positive attitude } \\
\text { toward swimming }\end{array}$ & & & $6 m$ & 1 & & & 1 & $\mathrm{n} / \mathrm{a}$ \\
\hline $\begin{array}{l}\text { Positive attitude } \\
\text { toward soccer and } \\
\text { handball }\end{array}$ & & & $7 f, 7 m$ & 2 & & & 2 & $\mathrm{n} / \mathrm{a}$ \\
\hline $\begin{array}{l}\text { Positive attitude } \\
\text { toward aerobic } \\
\text { fitness }\end{array}$ & & & $7 f, 7 m$ & 2 & & & 2 & $\mathrm{n} / \mathrm{a}$ \\
\hline \multicolumn{9}{|l|}{ Objective measurements } \\
\hline \multicolumn{9}{|l|}{ Accelerometer } \\
\hline PA volume & & & $52 f$ & 1 & & & 1 & $\mathrm{n} / \mathrm{a}$ \\
\hline PA intensity & $52 f$ & + & & 1 & 1 & & & $\mathrm{n} / \mathrm{a}$ \\
\hline Step count & $13 f$ & + & & 1 & 1 & & & $n / a$ \\
\hline $\begin{array}{l}\text { Moderate-to-vigorous } \\
\text { PA (continuous) }\end{array}$ & $13 f, 18,42 f, 42 m$ & + & & 4 & 4 & & & + \\
\hline $\begin{array}{l}\text { Vigorous PA } \\
\text { (continuous) }\end{array}$ & $13 f$ & + & & 1 & 1 & & & $\mathrm{n} / \mathrm{a}$ \\
\hline $\begin{array}{l}\text { Vigorous PA (none } \\
\text { vs. any) }\end{array}$ & 18 & & & 1 & 1 & & & $n / a$ \\
\hline Sedentary PA & $42 f, 42 m$ & - & & 2 & & 2 & & $\mathrm{n} / \mathrm{a}$ \\
\hline \multicolumn{9}{|l|}{ Physical fitness } \\
\hline $\begin{array}{l}\text { Knee extension } \\
\text { torque }\end{array}$ & & & 9 & 1 & & & 1 & $\mathrm{n} / \mathrm{a}$ \\
\hline Handgrip strength & & & 9 & 1 & & & 1 & $\mathrm{n} / \mathrm{a}$ \\
\hline Bench press & $7 m$ & + & $7 f$ & 2 & 1 & & 1 & $\mathrm{n} / \mathrm{a}$ \\
\hline Sargent jump & $6 f$ & + & & 1 & 1 & & & $n / a$ \\
\hline
\end{tabular}


Table 2 Summary of individual correlates and determinants of cardiorespiratory fitness (Continued)

\begin{tabular}{|c|c|c|c|c|c|c|c|c|}
\hline & \multicolumn{2}{|l|}{ Related to CRF } & \multirow{2}{*}{$\begin{array}{l}\text { Unrelated to CRF } \\
\text { Reference numbers }\end{array}$} & \multicolumn{5}{|c|}{ Summary of associations } \\
\hline & Reference numbers & $\begin{array}{l}\text { Association } \\
(+/-)\end{array}$ & & $\#$ & $\#+$ & $\#-$ & $\# 0$ & $\begin{array}{l}\text { Association } \\
(+/-)\end{array}$ \\
\hline \multicolumn{9}{|l|}{ Other } \\
\hline $\begin{array}{l}\text { Physical activity } \\
\text { energy expenditure }\end{array}$ & 24 & + & & 1 & & & & $\mathrm{n} / \mathrm{a}$ \\
\hline \multicolumn{9}{|l|}{ Other health behaviors } \\
\hline \multirow[t]{2}{*}{$\begin{array}{l}\text { Smoking vs. non- } \\
\text { smoking }\end{array}$} & $\begin{array}{l}8 f, 8 m, 11 f, 11 m, 22,23 m, 30 m, 32 m,(37 f, 63 f), 37 m \\
55 m, 60 m^{22}, 60 f^{2}\end{array}$ & - & \multirow{2}{*}{$\begin{array}{l}14 \mathrm{~m}, 16 \mathrm{f}, 16 \mathrm{~m}, 24 \\
30 \mathrm{f}, 35 \mathrm{~m},\left(12 \mathrm{f}^{19}\right. \\
\left.59 \mathrm{f}^{21}\right),\left(12 \mathrm{~m}^{19}\right. \\
\left.59 \mathrm{~m}^{21}\right)\end{array}$} & \multirow[t]{2}{*}{22} & \multirow[t]{2}{*}{1} & \multirow[t]{2}{*}{13} & \multirow[t]{2}{*}{8} & \multirow[t]{2}{*}{-} \\
\hline & $(14 f, 67)^{41}$ & + & & & & & & \\
\hline Number of cigarettes & $56 m^{20}, 68 m^{23}$ & - & $68 f$ & 3 & & 2 & 1 & - \\
\hline Alcohol & $12 f, 57^{24}$ & + & $12 m^{27}$ & 3 & 2 & & 1 & + \\
\hline Carbohydrates (g/day) & $47 m$ & + & & 1 & 1 & & & $\mathrm{n} / \mathrm{a}$ \\
\hline Diet quality score & $57 f, 57 m^{35}$ & + & & 2 & 2 & & & $\mathrm{n} / \mathrm{a}$ \\
\hline Meat dietary pattern & $57 \mathrm{f}^{26}, 57 \mathrm{~m}^{26}$ & - & & 2 & & 2 & & $\mathrm{n} / \mathrm{a}$ \\
\hline $\begin{array}{l}\text { Fruit-vegetable dietary } \\
\text { pattern }\end{array}$ & $57 m^{28}$ & + & $57 f$ & 2 & 1 & & 1 & $\mathrm{n} / \mathrm{a}$ \\
\hline $\begin{array}{l}\text { Childhood television } \\
\text { viewing }\end{array}$ & $(31,46)$ & - & & 1 & & 1 & & $\mathrm{n} / \mathrm{a}$ \\
\hline Adult television viewing & $(31,46)$ & - & & 1 & & 1 & & $\mathrm{n} / \mathrm{a}$ \\
\hline Sleep problems & $62^{25}$ & - & & 1 & & 1 & & $n / a$ \\
\hline \multicolumn{9}{|l|}{ Other } \\
\hline Commuting distance & $34^{34}$ & - & & 1 & & 1 & & $\mathrm{n} / \mathrm{a}$ \\
\hline $\begin{array}{l}\text { Gestational age } \\
\text { (mother) }\end{array}$ & $64 m$ & + & & 1 & 1 & & & $\mathrm{n} / \mathrm{a}$ \\
\hline $\begin{array}{l}\text { Attachment loss } \\
\text { (dental) }\end{array}$ & & & 67 & 1 & & & 1 & $\mathrm{n} / \mathrm{a}$ \\
\hline Probing depth (dental) & & & 67 & 1 & & & 1 & $\mathrm{n} / \mathrm{a}$ \\
\hline Preterm birth & & & 71 & 1 & 1 & & & $\mathrm{n} / \mathrm{a}$ \\
\hline
\end{tabular}

+: positive association; -: negative association; 0 : null association; $n / a$ : summary measure not applicable because the number of independent samples investigating the relationship is less than three. The numbers in the summary table refer to the reference number for each study. f: women only; m: men only. Samples from studies with longitudinal designs are marked in italic. Separate studies drawing on the same study population and reporting redundant exposures are presented in parentheses and were counted as one unit of analysis. CRF cardiorespiratory fitness, MET metabolic equivalent, LTPA leisure-time physical activity, PA physical activity, PWC physical working capacity 'Inverse U-shaped association

${ }^{2}$ Negatively associated with annual change in $\mathrm{VO}_{2 \max }$

${ }^{3}$ Significant association with test duration but not with work load 130

${ }^{4}$ Significant association with time to heart rate 130 but not with test duration

${ }^{5}$ Significant association with test duration but not with time to HR130

${ }^{6}$ Inverse U-shaped association (medium $>$ high $>$ low)

${ }^{7}$ Significant association with test duration but not with WL130

${ }^{8}$ Women with low CRF showed significantly higher waist circumference, compared with women with high CRF, in [77] (adjusted for "race" and age). Using the same data, in [14], women with high waist circumference did not show significantly different levels of CRF, compared with women with normal WC, after adjustment for multiple variables

${ }^{9}$ Lower $\mathrm{VO}_{2 \text { peak }}$ in the second quintile than in the third quintile of thyroid-stimulating hormone

${ }^{10}$ Lower odds of high fitness $\left(\mathrm{VO}_{2 \max }\right)$ with elevated serum ferritin $(>300 \mathrm{ng} / \mathrm{ml}) \mathrm{vs}$. non-elevated serum ferritin $(<300 \mathrm{ng} / \mathrm{ml})$

${ }^{11}$ Significant association with PWC150/kg but not with PWC150, workload/heart rate, test duration, or workload

${ }^{12}$ Significant association with workload and workload/heart rate but not with PWC150, PWC150/kg, or test duration

${ }^{13}$ Higher METS with medium vs. low financial strain

${ }^{14} \mathrm{Higher}$ mean $\mathrm{VO}_{2 \max }$ among white-collar workers than among blue-collar workers and farmers

${ }^{15}$ Significant positive association with time to heart rate 130; significant negative association with test duration

${ }^{16}$ Significant association with workload, workload/heart rate, PWC150, and test duration but not with PWC150/kg

${ }^{17}$ Significant negative association with test duration only for black women; significant positive association with WL130 only for white women

${ }^{18}$ Significant association with exercise test duration only for white men (not for black men)

${ }^{19}$ Significant negative association with test duration; significant positive association with time to heart rate 130

${ }^{20}$ Significant negative association with workload, test duration, and workload/heart rate; no significant association with PWC150; significant positive association with PWC150/kg 


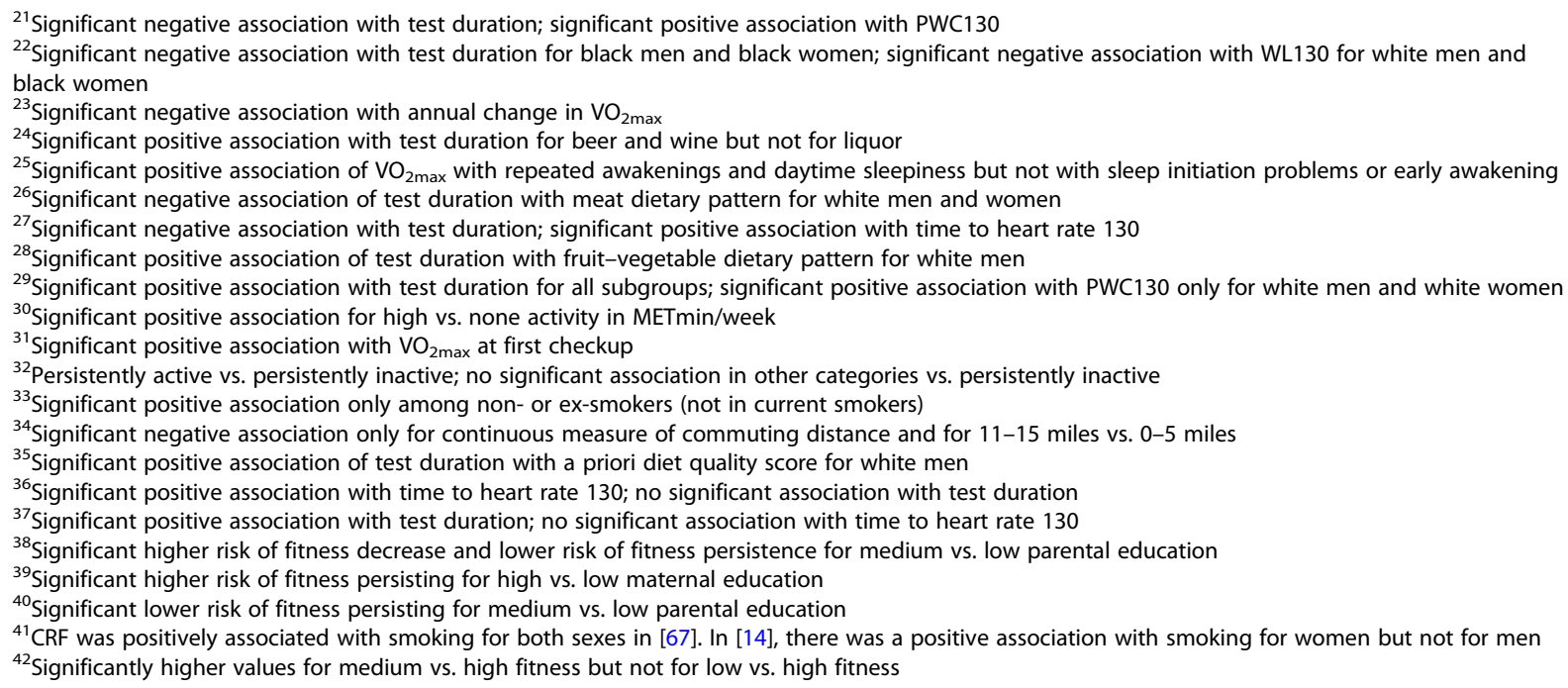

\section{Vital Parameters}

Among studies investigating the relationship between vital parameters and $\mathrm{CRF}$, resting $\mathrm{HR}$, systolic $\mathrm{BP}$, forced expiratory volume (FEV) in $1 \mathrm{~s}$, and vital capacity were each studied in three or more samples. Resting HR and systolic BP showed negative associations with CRF, whereas vital capacity and FEV in $1 \mathrm{~s}$ were positively associated with CRF.

\section{Biomarkers}

A variety of biomarkers were investigated in a total of 22 samples. Most clearly, CRP showed a negative association with CRF. High-density lipoprotein (HDL) cholesterol was found to be positively associated with CRF in three samples. Other biomarkers were studied in fewer than three samples.

\section{Activity Parameters}

About half of the studies (39) reported at least one measure of PA. A wide range of PA measures were used, but only five of these measures were reported in at least three independent samples, allowing the calculation of a summary measure. Most studies investigating activity parameters assessed subjective PA (e.g., via the International Physical Activity Questionnaire; 34), but objective activity parameters (using an accelerometer or objective physical fitness measures) were collected in four studies. Most studies assessing subjective PA used measures of overall PA, namely customized PA indexes, dichotomous PA level (high vs. low), PA times per week, or PA in metabolic equivalents/metabolic equivalent minutes. Forms of LTPA were also often investigated, but these were too diverse for the calculation of summary measures. Other specific domains of PA (transport PA, occupational PA, or household PA) and sedentary behavior were rarely or never examined. Moderate-to- vigorous PA was the only objective measure of PA (measured using an accelerometer) reported in at least three independent samples. Almost all summarized PA exposures showed a positive association with CRF. Only dichotomous PA level (high vs. low) showed no association (based on three samples).

\section{Other Behavioral Factors}

Other than PA, smoking status was the most frequently investigated indicator of health behavior (22 samples). Overall, smoking was inversely associated with CRF. However, in a considerable number of samples, smoking status was found to be unrelated to CRF, and one study found a positive association between smoking and CRF in women. The number of cigarettes consumed, which was studied in three samples, was also inversely associated with CRF.

Alcohol consumption was examined in three samples. Whereas one mixed-sex sample showed a positive association between alcohol consumption and CRF, another study found a positive association among women but varying results depending on the CRF measure among men.

For all other investigated behavioral factors, the results could not be summarized because of a limited number of samples.

\section{Sensitivity Analyses}

No significant differences in the association with CRF were found for age, education, or WC when comparing different methods of CRF assessment (direct $\mathrm{VO}_{2 \max }$ vs. indirect $\mathrm{VO}_{2 \max }$ vs. other), different test machines used (bicycle ergometer vs. treadmill) or sex (men vs. women vs mixed samples; Additional file 2: Tables OR2.1 to OR2.19). For BMI, PA index, and smoking, we found differences in the results by the CRF measure used $(p<$ 
0.05 , Fisher's exact test) but not by sex and test device. The association between CRF and BMI did not differ between samples where adjustments for body weight measures were performed, compared with samples where this adjustment was not performed.

\section{Update Literature Search}

The limited update literature search in PubMed yielded 383 records. After de-duplicating for records found in the initial search and title and abstract screening, 55 records remained for full-text screening. A total of seven records did fulfil the eligibility criteria [158-164]. Study characteristics and results of these studies can be found in Additional file 3: Table OR3.1. Overall, results from these studies are in line with the reported results from the main search and only few new potential correlates or determinants were investigated: one study analyzing caffeine consumption found a positive association with CRF among women, but not among men [162]. Another study reported a negative association between various plasma fatty acids and CRF among men, but only between arachidonic acid and CRF among women [163]. Moreover, in a further study, a negative association between anemia and CRF among women and a negative association between estimated glomerular filtration rate and CRF among men was reported [161]. As these factors were reported in two or less samples, calculation of a summary measure was not possible.

\section{Discussion}

\section{Summary of Evidence}

This systematic review aimed to give a detailed overview of the potential individual factors influencing CRF and to analyze the consistency of the results of existing research on this topic. Overall, 3016 records were identified, and 78 articles were ultimately included. We found evidence that CRF decreases with age, is lower among women than among men, and is associated with ethnicity. CRF was positively associated with SES, FEV, and vital capacity, and negatively associated with BMI, weight, WC, body fat, resting HR, systolic BP, and CRP. As expected, CRF was associated with several measures of PA. Furthermore, CRF showed a negative association with smoking and a positive association with alcohol consumption in the majority of the included studies. Age, BMI, WC, PA index, smoking, and education were the most investigated factors ( $\geq 10$ samples). For these factors, results showed no significant sex differences. To our knowledge, this is the first comprehensive review investigating the individual determinants and correlates of CRF. A review conducted by Ortega et al. [98] summarized the relationship between fitness and CVD risk factors among children and adolescents. Another systematic review focused on studies investigating the association between genetic variants and CRF trainability [113]; however, this was beyond the scope of the present review.

\section{Comparison with Other Studies and Interpretation of Results \\ Sociodemographic Characteristics}

It is well established that CRF declines with age. This may be because of physiological adjustments, such as atrophy of muscle mass with biological aging, changes in lifestyle, or increasing disease burden and medication [118]. Although the decline in CRF with age is widely accepted, the causes behind this relation are not yet clearly understood [119].

The contradictory results for age found in two included samples can partially be explained by the limited age ranges of these study samples: The studies focused on young or middle-aged individuals. Because maximum CRF is usually attained between 20 and 30 years of age [37, 86, 120], linear age trends may be positive in young study populations. When the study population has an older age range, a decline in CRF with age becomes apparent-a trend that seems to be more pronounced among men than among women [22, 37, 120, 121]. However, these differences largely disappear when the decline in CRF is expressed as a percentage change rather than as an absolute value [37, 121]. Two studies used meta-analytical approaches to combine data and generate general agespecific reference values and predictive equations [99, 122]. However, because of a high level of heterogeneity in the assessment of CRF, as well as differences in methods and study population characteristics, these results should be interpreted with caution [123]. Furthermore, the effect of PA on the decline in CRF over the life course is unclear $[120,121]$. Although metaanalyses of cross-sectional data have not reported evidence that increased PA levels mitigate the decline [124, 125], longitudinal studies have found that individuals with enhanced PA have less decline in CRF per decade than do sedentary individuals [120]. Independent of age, a longitudinal study among men found a greater decline in CRF over time associated with a greater risk of total mortality [157].

There is clear evidence that, overall, compared with men, women have lower CRF levels. The difference between men and women is often estimated at around 20\% [20, 126-128]. Common physiological explanations for this difference are women's smaller body and organ size and higher body fat percentage, compared with men [100]. Correspondingly, Fleg et al. [22] pointed out that, when $\mathrm{VO}_{2 \max }$ is expressed relative to muscle mass (e.g., $\mathrm{ml} / \mathrm{kg}$ muscle mass $/ \mathrm{min}$ ), the difference between the sexes is often eliminated. In addition to physiological explanations, there are differences in behavioral and social 
factors [100] (e.g., differences in PA behavior) that could partly explain the CRF differences between men and women.

Higher SES is predominantly associated with higher CRF. Although our summary measures could only be calculated for education and composite SES indicators, no inverse association was reported for the other SES indicators, which were investigated in fewer than three samples. A positive association between education and CRF was previously confirmed in a meta-analysis that included 9435 adults from four population-based studies [102]. These findings are also in line with systematic reviews investigating the association between LTPA and CRF [129]. Because aerobic LTPA is strongly associated with CRF, lower LTPA levels among lower-SES population groups may contribute to lower CRF levels in these groups. Lower SES is not only associated with lower aerobic LTPA but also with higher fat and sugar consumption, lower consumption of fruits and vegetables [130], and higher smoking prevalence [131]. In addition to unfavorable health behaviors, major NCD risk factors like obesity [132] and chronic diseases such as diabetes and CVD are more prevalent among population groups with lower SES. Thus, unfavorable health behaviors and risk factors that may be associated with CRF potentially reinforce the effect of SES on CRF. However, these associations have been confirmed mainly in high- and upper-middle-income countries, and the situation in low- or middle-income countries might be different because these countries are in earlier stages of the epidemiological transition [133]. High-SES population groups in these countries may move to the next stage before low-SES groups and therefore also adopt unfavorable health behaviors earlier. Consider, for example, the case of obesity: In low-income countries, the more affluent are more likely to be obese [134].

\section{Anthropometric Measures and Vital Parameters}

Anthropometric measures were often investigated in the included studies, showing a clear inverse association between BMI and CRF. Most samples with an inverse association between CRF and BMI used a body weightadjusted measure of CRF (such as relative $\mathrm{VO}_{2 \max }$ in $\mathrm{ml} /$ $\mathrm{kg} / \mathrm{min}$ ) that took into account the differences in CRF because of BMI. We performed sensitivity analyses to check whether the association between CRF and BMI differed depending on the adjustment of measures for relative body weight and found no significant differences. Although CRF and BMI are related, a high BMI does not necessarily mean a low fitness level. High muscle mass can also lead to having a high BMI caused by the dense structure of muscle mass. Research suggests that, compared with the health risks of having a high BMI, having low CRF is a more important risk factor: a systematic review found that the risk for cardiovascular mortality was lower among individuals with high BMIs and high levels of CRF, compared with those with normal BMIs and low levels of CRF [135]. Furthermore, studies suggest that individuals with low CRF have higher levels of general and abdominal adiposity, as measured by WC, for example, than do individuals with moderate/high CRF, independent of BMI [136]. Thus, it is not surprising that WC was negatively associated with CRF in almost all of the included studies. Likewise, percentage of body fat was found to be negatively associated with CRF in all samples, and lean body mass (or skeletal muscle mass, which accounts for most of the lean mass) was positively associated with CRF. Again, it should be noted that the effects of these factors are strongly depending on the application and procedures (e.g., relative to body weight, relative to fat free mass, or relative to predicted weights) of data normalization for the CRF measure [165].

All of the reviewed studies demonstrated the expected associations between CRF and vital capacity parameters, most of which have been extensively studied in endurance training intervention studies and well documented in the exercise physiology literature [137]. $\mathrm{VO}_{2 \max }$ is one of many physiological parameters that improve as a result of endurance training. Increased cardiorespiratory capacities such as stroke volume and vital capacity, as well as improved muscle and blood composition, contribute to a higher maximal oxygen uptake. These adaptations lead to a more efficient supply of energy and oxygen for bodily functions and to a reduction in resting $\mathrm{HR}$ and BP.

\section{Behavioral Factors}

The majority of the included studies found a negative association between CRF and smoking. These findings may be explained by multiple pathways. First, tobacco smoking may trigger a cascade of modifications in the respiratory organs, which can lead to reduced pulmonary function. Limited pulmonary function, in turn, may be negatively associated with CRF [138]. Second, because CRF and PA are closely related and PA is also inversely associated with smoking, negative health behaviors such as inactivity, smoking, and alcohol consumption may often cluster together [139], and (in)activity acts as a confounder in the relation between smoking and CRF. Third, the relation between CRF and smoking also may be explained by SES because both smoking [131] and low fitness [102] seem to be associated with low SES.

Because the observed association of CRF with alcohol is based on only three samples, these findings should be interpreted with caution. One study found a direct association with some types of alcohol (e.g., wine) but not others (e.g., liquor) [57]. A recent investigation found 
better fitness among moderate drinkers, compared with heavy drinkers and abstainers, using data from five population-based studies [101]. Although there are potential explanations for the U-shaped relation between alcohol intake and CRF [101], it is also possible that this is a result of confounding [140].

Dietary risk factors, such as a diet high in sugar-sweetened beverages, are known to be related to cardiometabolic risk factors [141], but relatively little is known regarding the relationship between these dietary factors and CRF [57, 70]. Recent research suggests that CRF may act as a mediator of the relation between macronutrient intake relative to weight and fat mass in adolescents: individuals with a higher macronutrient intake, which may be related to engaging in more PA, may suffer from obesity less often, especially when they have high CRF levels [142].

The relation between PA and CRF has been widely discussed in the literature, and the positive impacts of PA and CRF on health are well documented [143]. CRF is influenced by several physiological traits, especially the performance of the cardiovascular system. Thus, in the relationship between PA and CRF, PA is the modifiable variable: performing $\mathrm{PA}$ allows individuals to reach their highest possible level of CRF, which is also determined by heredity and other factors $[84,144]$. Although the nature of the association between PA and CRF is widely accepted, it cannot be ruled out that low CRF levels caused by genetic pre-conditions can lead to lower PA levels [84].

The results of this systematic review reflect the wellestablished relation between CRF and PA. A wide variety of objective and subjective PA measures were used in the included studies, and a majority of these studies found a significant positive association between PA and CRF. Whereas overall PA and LTPA were commonly investigated, only a few studies investigated other domains such as occupational PA. Other domain-specific types of PA may have different effects on health. For example, studies have shown that occupational PA can either have no association or be negatively associated with health outcomes, whereas LTPA affects health in a strictly beneficial way $[145,146]$. While conducting this review, we found only two studies that investigated the relation between PA at work and CRF, and these studies had varying results. One study found no association between CRF and occupational PA but a significant positive association between CRF and hours of PA per week [45]. The other study found a positive association for both LTPA and occupational PA [74]. Considering their potentially varying effects on health, the associations between domain-specific types of PA and CRF should be further examined.

Overall, health habits such as dietary, smoking, and PA behaviors are usually measured with considerable inaccuracy. This could mean that there is imprecision in the measurement of health behaviors in studies investigating these factors as correlates and determinants of CRF [47].

\section{Strengths and Limitations}

To our knowledge, this is the first systematic review investigating the individual correlates and determinants of CRF. The semi-quantitative approach used in this review made it possible to present a concise summary of the results from 78 studies and to provide a comprehensive overview of the current state of research and potential research gaps.

We acknowledge that such a comprehensive review also has limitations. The majority of the studies included in this review were cross-sectional; therefore, statements about causal relationships between exposures and outcomes are limited. Furthermore, not all studies used $\mathrm{VO}_{2 \max }$ (the "gold standard") as an outcome, diminishing the comparability of results across the studies. Although other methods for measuring CRF (e.g., exercise test duration) have been shown to be correlated with $\mathrm{VO}_{2 \max }[147,148]$, differences in methods could lead to deviating results. It is interesting to note that the included studies that reported the associations between an exposure and various measures of CRF often found similar associations, independent of the measurement method used. Several studies also obtained CRF results using submaximal exercise-based predictive equations. Although it has been found that the results of assessed and predicted CRF can vary [111], a systematic review has demonstrated that these predictive equations are quite accurate [149]. In addition to differences in the method of measurement (indirect vs. direct), different indicators of $\mathrm{CRF}$ (e.g., $\mathrm{VO}_{2 \max }$ vs. others) were not taken into account when discussing the results. This could be considered a potential bias because sensitivity analyses showed significant differences in the association between directly assessed $\mathrm{VO}_{2 \max }$, indirectly assessed $\mathrm{VO}_{2 \max }$, and other CRF measures for some of the exposures. Variations in the methods used in the statistical analyses may also have affected the results. In this systematic review, we only analyzed the adjusted results from multivariable analyses. Because fewer variables usually reach the defined level of significance in such analyses, compared with univariate analyses [115], a bias toward null findings is possible [150]. Such a bias may be exacerbated by the use of the fully adjusted models.

Because of the wide range of correlates and determinants analyzed, as well as the significant heterogeneity in analytical methods and study samples, it was not feasible to conduct a meta-analysis. Correspondingly, we were unable to show the strength of the associations (effect measures) or to adjust the results for sample size. In 
addition, variation in adjustment for confounding among the studies was not considered in the interpretation of the results for each outcome, although this difference may have led to divergent results across the studies. Due to restrictions in the search strategy and the intra-study requirements to participate in CRF testing, the study populations usually consist of a relatively healthy population. However, it cannot be ruled out that some of the reported associations are influenced by underlying chronic diseases. Furthermore, the universal perspective of this review did not allow determinants, correlates, mediators, moderators, and confounders to be differentiated [151]. However, the focus of this review was to show the consistency of reported associations. Certain indicators may be underrepresented for specific reasons (e.g., underreporting or limitations of the search strategy). Existing studies have shown that correlates and determinants may differ across the life course [37, 98]. Future studies should investigate whether the results of this systematic review can be confirmed for young people and older adults. Furthermore, although this was beyond the scope of the present review, environmental and interpersonal determinants probably also play an important role in determining or moderating CRF and should be further investigated [103].

\section{Conclusions}

Our comprehensive systematic review showed that there is a broad range of individual factors associated with CRF. Whereas factors such as age, BMI, and WC showed consistent evidence of an association with CRF, other factors showed conflicting or insufficient evidence of an association. For example, few studies have investigated the relationship between CRF and behavioral factors other than PA and smoking, or the association between CRF and psychosocial factors.

Several implications for health promotion practice and for future research can be drawn from this review. First, sociodemographic factors shown to be associated with CRF in this review can be used to help identify subgroups of relatively unfit individuals (e.g., people with low education) who should be targeted for interventions. Second, the strong association between aerobic LTPA and CRF was confirmed in this review, but less is known about the relation between CRF and domain-specific PA. Thus, future studies should compare the impact of domain-specific PA on CRF. The comparability of the results was hampered by differences in the assessment of $\mathrm{PA}$; therefore, the use of standardized domain-specific measures, such as the Global Physical Activity Questionnaire [152] or the European Health Interview SurveyPhysical Activity Questionnaire [153], is recommended. In addition, sedentary behavior plays an important role in the global strategy to tackle NCDs, but little is known about the association of this factor with CRF in adults [154]. Third, there is some evidence that health behaviors other than PA, such as smoking, alcohol consumption, and nutrition, are also associated with CRF. Thus, multi-behavioral interventions might be an appropriate approach when implementing preventive measures to enhance health status in the general adult population [155], although the effects of these approaches have been found to be limited [156].

Future reviews could build on the results of the present systematic review by consolidating the evidence regarding the correlates and determinants of CRF in younger and older population groups, which were not included in this review. Moreover, evidence from lowand middle-income countries is needed to improve the generalizability of the results because most of the studies included in this review were conducted in high-income country settings. Notwithstanding the close association between CRF and PA and the fact that CRF is partly genetically determined, relatively little is known about the complex interplay among the potential determinants of CRF. This review can be a first step toward the development of a comprehensive model of (cardiorespiratory) fitness that integrates not only physiological aspects but also a broad set of socio-ecological factors.

\section{Additional Files}

Additional file 1: Quality Assessment. (PDF $490 \mathrm{~kb}$ )

Additional file 2: Sensitivity Analyses. (PDF $253 \mathrm{~kb}$ )

Additional file 3: Limited Search Update. (PDF $210 \mathrm{~kb}$ )

Additional file 4: Data extraction table. (XLSX $215 \mathrm{~kb}$ )

\section{Abbreviations}

BMI: Body mass index; BP: Blood pressure; CRF: Cardiorespiratory fitness; CRP: C-reactive protein; CVD: Cardiovascular disease; HDL: High-density lipoprotein; HR: Heart rate; LTPA: Leisure-time physical activity; NCD: Noncommunicable disease; PA: Physical activity; PRISMA: Preferred Reporting Items for Systematic Reviews and Meta-Analyses; PROSPERO: International Prospective Register of Systematic Reviews; SES: Socioeconomic status; $\mathrm{VO}_{2 \text { max }}$ : Maximal oxygen consumption; $\mathrm{VO}_{2 \text { peak: }}$ Peak oxygen consumption; WC: Waist circumference

Acknowledgment

We thank Jennifer Barrett for editing a draft of this manuscript.

\section{Authors' Contributions}

JZ, NP, TK, GBMM, and JDF contributed to the conception and design of the study. JZ, KJO, NP, and JDF contributed to the acquisition, analysis, and assembly of the data. JZ, KJO, GBMM, and JDF contributed to the interpretation of the data. JZ wrote the first draft and final version of the manuscript. KJO and JDF contributed to the writing of the manuscript. All of the authors critically revised the manuscript for important intellectual content and approved the final manuscript. Additionally, all of the authors agree to be accountable for all aspects of the work in ensuring that questions related to the accuracy or integrity of any part of the work are appropriately investigated and resolved. 


\section{Funding}

No external funding was received. The study was financed by the Robert Koch Institute, which is a federal institute within the portfolio of the German Federal Ministry of Health.

\section{Availability of Data and Materials}

All data generated or analyzed during this study are included in this published article (and its supplementary information files).

\section{Ethics Approval and Consent to Participate}

Not applicable.

\section{Consent for Publication}

Not applicable.

\section{Competing Interests}

The authors, Johannes Zeiher, Katherine J. Ombrellaro, Nita Perumal, Thomas Keil, Gert B. M. Mensink, and Jonas D. Finger, declare that they have no competing interests.

\section{Author details}

'Department of Epidemiology and Health Monitoring, Robert Koch Institute, General-Pape-Straße 62-66, 12101 Berlin, Germany. ${ }^{2}$ Department of Infectious Disease Epidemiology, Robert Koch Institute, Berlin, Germany. Institute for Clinical Epidemiology and Biometry, University of Würzburg, Würzburg, Germany. ${ }^{4}$ Institute for Health Resort Medicine and Health Promotion, Bavarian Health and Food Safety Authority, Bad Kissingen, Germany. ${ }^{5}$ Institute for Social Medicine, Epidemiology and Health Economics, Charité Universitätsmedizin Berlin, Berlin, Germany.

\section{Received: 24 April 2019 Accepted: 5 August 2019}

Published online: 03 September 2019

\section{References}

1. Abramowitz MK, Hostetter TH, Melamed ML. Lower serum bicarbonate and a higher anion gap are associated with lower cardiorespiratory fitness in young adults. Kidney Int. 2012;81:1033-42. https://doi.org/10.1038/ki.2011.479.

2. Agarwal S. Red cell distribution width, inflammatory markers and cardiorespiratory fitness: results from the National Health and Nutrition Examination Survey. Indian Heart J. 2012;64:380-7.

3. Allen JG. Aerobic capacity and physiological fitness of Australian men. Ergonomics. 1966;9:485-94

4. Ardestani A, Parker B, Mathur S, Clarkson P, Pescatello LS, Hoffman HJ, et al. Relation of vitamin D level to maximal oxygen uptake in adults. Am J Cardiol. 2011;107:1246-9. https://doi.org/10.1016/.jamjcard.2010.12.022.

5. Babcock MA, Paterson DH, Cunningham DA. Influence of ageing on aerobic parameters determined from a ramp test. Eur J Appl Physiol. 1992;65:138-43.

6. Barnekow-Bergkvist M, Hedberg G, Janlert U, Jansson E. Prediction of physical fitness and physical activity level in adulthood by physical performance and physical activity in adolescence -an 18-year follow-up study. Scand J Med Sci Sports. 1998;8:299-308.

7. Barnekow-Bergkvist M, Hedberg G, Janlert U, Jansson E. Adolescent determinants of cardiovascular risk factors in adult men and women. Scand J Public Health. 2001;29:208-17.

8. Bernaards CM, Twisk JW, Van Mechelen W, Snel J, Kemper HC. A longitudinal study on smoking in relationship to fitness and heart rate response. Med Sci Sports Exerc. 2003;35:793-800. https://doi.org/10.1249/01. mss.0000064955.31005.e0.

9. Bijlsma AY, Meskers CG, van den Eshof N, Westendorp RG, Sipila S, Stenroth $L$, et al. Diagnostic criteria for sarcopenia and physical performance. Age. 2014;36:275-85. https://doi.org/10.1007/s11357-013-9556-5.

10. Binder J, Bailey KR, Seward JB, Squires RW, Kunihiro T, Hensrud DD, et al. Aortic augmentation index is inversely associated with cardiorespiratory fitness in men without known coronary heart disease. Am J Hypertens. 2006;19:1019-24.

11. Blair SN, Kannel WB, Kohl HW, Goodyear N, Wilson PW. Surrogate measures of physical activity and physical fitness: evidence for sedentary traits of resting tachycardia, obesity, and low vital capacity. Am J Epidemiol. 1989; 129:1145-56.

12. Braun $\mathrm{BL}$, Wagenaar $\mathrm{AC}$, Flack JM. Alcohol consumption and physical fitness among young adults. Alcohol Clin Exp Res. 1995;19:1048-54.
13. Cao Z, Miyatake N, Higuchi M, Miyachi M, Ishikawa-Takata K, Tabata I. Predicting $\mathrm{VO}_{2 \max }$ with an objectively measured physical activity in Japanese women. Med Sci Sports Exerc. 2010;42:179-86.

14. Ceaser TG, Fitzhugh EC, Thompson DL, Bassett DR Jr. Association of physical activity, fitness, and race: NHANES 1999-2004. Med Sci Sports Exerc. 2013; 45:286-93. https://doi.org/10.1249/MSS.0b013e318271689e.

15. Cleland VJ, Ball K, Magnussen C, Dwyer T, Venn A. Socioeconomic position and the tracking of physical activity and cardiorespiratory fitness from childhood to adulthood. Am J Epidemiol. 2009;170:1069-77. https://doi. org/10.1093/aje/kwp271.

16. Dagan SS, Segev S, Novikov I, Dankner R. Waist circumference vs body mass index in association with cardiorespiratory fitness in healthy men and women: a cross sectional analysis of 403 subjects. Nutr J. 2013;12:12. https:// doi.org/10.1186/1475-2891-12-12.

17. Dehn MM, Bruce RA. Longitudinal variations in maximal oxygen intake with age and activity. J Appl Physiol. 1972;33:805-7.

18. Dyrstad SM, Anderssen SA, Edvardsen E, Hansen BH. Cardiorespiratory fitness in groups with different physical activity levels. Scand J Med Sci Sports. 2016;26:291-8. https://doi.org/10.1111/sms.12425.

19. Farrell SW, Kohl HW, Rogers T. The independent effect of ethnicity on cardiovascular fitness. Hum Biol. 1987;59:657-66.

20. Finger JD, Krug S, Gosswald A, Hartel S, Bos K. Kardiorespiratorische Fitness bei Erwachsenen in Deutschland: Ergebnisse der Studie zur Gesundheit Erwachsener in Deutschland (DEGS1) [Cardiorespiratory fitness among adults in Germany: results of the German Health Interview and Examination Survey for Adults (DEGS1)]. Bundesgesundheitsblatt Gesundheitsforschung Gesundheitsschutz. 2013;56:772-8. https://doi.org/10.1007/s00103-013-1672-y.

21. Fleg JL, Lakatta EG. Role of muscle loss in the age-associated reduction in $V O_{2}$ max. J Appl Physiol. 1988;65:1147-51.

22. Fleg JL, Morrell CH, Bos AG, Brant LJ, Talbot LA, Wright JG, et al. Accelerated longitudinal decline of aerobic capacity in healthy older adults. Circulation. 2005;112:674-82.

23. Fogelholm M, Malmberg J, Suni J, Santtila M, Kyröläinen H, Mäntysaari M. Waist circumference and BMI are independently associated with the variation of cardio-respiratory and neuromuscular fitness in young adult men. Int J Obes. 2006;30:962-9.

24. Franks PW, Barroso I, Luan J, Ekelund U, Crowley V, Brage S, et al. PGC-1a genotype modifies the association of volitional energy expenditure with VO $_{2 \max }$. Med Sci Sports Exerc. 2003;35:1998-2004.

25. Gläser S, Friedrich N, Ewert R, Schäper C, Krebs A, Dörr M, et al. Association of circulating IGF-I and IGFBP-3 concentrations and exercise capacity in healthy volunteers: results of the Study of Health in Pomerania. Growth Horm IGF Res. 2010;20:404-10.

26. Gläser S, Obst A, Opitz CF, Dörr M, Felix SB, Empen K, et al. Peripheral endothelial dysfunction is associated with gas exchange inefficiency in smokers. Respir Res. 2011;12:53.

27. Grigaliuniene A, Ramonas A, Celutkiene J, Sileikiene V, Rudys A, Juocevicius $A$, et al. Cardiorespiratory parameters of exercise capacity in a healthy Lithuanian population: the pilot study. Hellenic J Cardiol. 2013;54:107-18.

28. Gulati M, Black HR, Shaw $\sqcup$, Arnsdorf MF, Merz CNB, Lauer MS, et al. The prognostic value of a nomogram for exercise capacity in women. N Engl J Med. 2005;353:468-75.

29. Habedank D, Reindl I, Vietzke G, Bauer U, Sperfeld A, Gläser S, et al. Ventilatory efficiency and exercise tolerance in 101 healthy volunteers. Eur J Appl Physiol. 1998;77:421-6.

30. Hakola L, Komulainen P, Hassinen M, Savonen K, Litmanen H, Lakka TA, et al. Cardiorespiratory fitness in aging men and women: the DR's EXTRA study. Scand J Med Sci Sports. 2011;21:679-87. https://doi.org/10.1111/j.16 00-0838.2010.01127.x.

31. Hancox RJ, Milne BJ, Poulton R. Association between child and adolescent television viewing and adult health: a longitudinal birth cohort study. Lancet. 2004;364:257-62.

32. Hawari Fl, Obeidat NA, Ghonimat IM, Ayub HS, Dawahreh SS. The effect of habitual waterpipe tobacco smoking on pulmonary function and exercise capacity in young healthy males: a pilot study. Respir Med. 2017;122:71-5.

33. Heil DP, Freedson PS, Ahlquist LE, Price J, Rippe JM. Nonexercise regression models to estimate peak oxygen consumption. Med Sci Sports Exerc. 1995; 27:599-606.

34. Hoehner CM, Barlow CE, Allen P, Schootman M. Commuting distance, cardiorespiratory fitness, and metabolic risk. Am J Prev Med. 2012;42:571-8. https://doi.org/10.1016/j.amepre.2012.02.020. 
35. Inbar O, Oren A, Scheinowitz M, Rotstein A, Dlin R, Casaburi R. Normal cardiopulmonary responses during incremental exercise in 20-to 70-yr-old men. Med Sci Sports Exerc. 1994;26:538-46.

36. Ittermann T, Gläser S, Ewert R, Felix S, Völzke H, Dörr M. Serum thyroidstimulating hormone levels are not associated with exercise capacity and lung function parameters in two population-based studies. BMC Pulm Med. 2014;14:145. https://doi.org/10.1186/1471-2466-14-145.

37. Jackson AS, Sui X, Hebert JR, Church TS, Blair SN. Role of lifestyle and aging on the longitudinal change in cardiorespiratory fitness. Arch Intern Med. 2009;169:1781-7. https://doi.org/10.1001/archinternmed.2009.312.

38. Kemper HC, Twisk JW, Koppes LL, van Mechelen W, Post GB. A 15-year physical activity pattern is positively related to aerobic fitness in young males and females (13-27 years). Eur J Appl Physiol. 2001;84:395-402. https://doi.org/10.1007/s004210100392.

39. Kim JK. Changes in cardiorespiratory fitness, body composition, and physical activity of women aged 20 to 68 years. Int J Hum Mov Sci. 2011;5:69-78.

40. Koch B, Schäper C, Ittermann T, Spielhagen T, Dörr M, Völzke H, et al. Reference values for cardiopulmonary exercise testing in healthy volunteers: the SHIP study. Eur Respir J. 2009;33:389-97.

41. Kondo N, Nomura M, Nakaya Y, Ito S, Ohguro T. Association of inflammatory marker and highly sensitive C-reactive protein with aerobic exercise capacity, maximum oxygen uptake and insulin resistance in healthy middleaged volunteers. Circ J. 2005;69:452-7.

42. Kulinski JP, Khera A, Ayers CR, Das SR, de Lemos JA, Blair SN, et al. Association between cardiorespiratory fitness and accelerometer-derived physical activity and sedentary time in the general population. Mayo Clin Proc. 2014;89:1063-71. https://doi.org/10.1016/j.mayocp.2014.04.019.

43. Kuo HK, Yen CJ, Bean JF. Levels of homocysteine are inversely associated with cardiovascular fitness in women, but not in men: data from the National Health and Nutrition Examination Survey 1999-2002. J Intern Med. 2005;258:328-35. https://doi.org/10.1111/j.1365-2796.2005.01546.x.

44. Kuo HK, Yen CJ, Chen JH, Yu YH, Bean JF. Association of cardiorespiratory fitness and levels of C-reactive protein: data from the National Health and Nutrition Examination Survey 1999-2002. Int J Cardiol. 2007;114:28-33. https://doi.org/10.1016/j.ijcard.2005.11.110.

45. Lakka TA, Kauhanen J, Salonen JT. Conditioning leisure time physical activity and cardiorespiratory fitness in sociodemographic groups of middle-aged men in eastern Finland. Int J Epidemiol. 1996;25:86-93.

46. Landhuis CE, Poulton R, Welch D, Hancox RJ. Programming obesity and poor fitness: the long-term impact of childhood television. Obesity. 2008;16: 1457-9. https://doi.org/10.1038/oby.2008.205.

47. Laukkanen JA, Laaksonen D, Lakka TA, Savonen K, Rauramaa R, Mäkikallio T, et al. Determinants of cardiorespiratory fitness in men aged 42 to 60 years with and without cardiovascular disease. Am J Cardiol. 2009;103:1598-604.

48. Lefevre J, Philippaerts R, Delvaux K, Thomis M, Claessens AL, Lysens R, et al. Relation between cardiovascular risk factors at adult age, and physical activity during youth and adulthood: the Leuven Longitudinal Study on Lifestyle, Fitness and Health. Int J Sports Med. 2002;23(Suppl 1):S32-8. https://doi.org/10.1055/s-2002-28459.

49. MacAuley D, Mc Crum EE, Stott G, Evans AE, Gamble RP, Mc Roberts B, et al. Levels of physical activity, physical fitness and their relationship in the Northern Ireland Health and Activity Survey. Int J Sports Med. 1998;19:50311. https://doi.org/10.1055/s-2007-971952.

50. Mainous AG 3rd, Diaz VA. Relation of serum ferritin level to cardiovascular fitness among young men. Am J Cardiol. 2009;103:115-8. https://doi.org/1 0.1016/j.amjcard.2008.08.046.

51. Makrides L, Heigenhauser GJ, McCartney N, Jones NL. Maximal short term exercise capacity in healthy subjects aged 15-70 years. Clin Sci. 1985;69:197-205.

52. Nokes N. Relationship between physical activity and aerobic fitness. J Sports Med Phys Fitness. 2009;49:136-41.

53. Plowman SA, Drinkwater BL, Horvath SM. Age and aerobic power in women: a longitudinal study. J Gerontol. 1979;34:512-20.

54. Sanada K, Kuchiki T, Miyachi M, McGrath K, Higuchi M, Ebashi H. Effects of age on ventilatory threshold and peak oxygen uptake normalised for regional skeletal muscle mass in Japanese men and women aged 20-80 years. Eur J Appl Physiol. 2007;99:475-83. https://doi.org/10.1007/s00421-006-0375-6.

55. Sandvik L, Erikssen G, Thaulow E. Long term effects of smoking on physical fitness and lung function: a longitudinal study of 1393 middle aged Norwegian men for seven years. BMJ. 1995;311:715.

56. Seccareccia F, Menotti A, Fazzini PF, Prati PL, Antoniucci D, Menghini F. Determinants of physical performance at cycloergometer in healthy middle aged men in Italy. The ECCIS project. Epidemiology and clinics of silent ischemic heart disease. Acta Cardiol. 1997;52:49-65.

57. Shikany JM, Jacobs DR Jr, Lewis CE, Steffen LM, Sternfeld B, Carnethon MR, et al. Associations between food groups, dietary patterns, and cardiorespiratory fitness in the Coronary Artery Risk Development in Young Adults study. Am J Clin Nutr. 2013;98:1402-9. https://doi.org/10.3945/ajen.113.058826.

58. Shmueli H, Rogowski O, Toker S, Melamed S, Leshem-Rubinow E, Ben-Assa E, et al. Effect of socioeconomic status on cardio-respiratory fitness: data from a health screening program. J Cardiovasc Med. 2014;15:435-40. https://doi.org/10.2459/JCM.0b013e3283629c87.

59. Sidney S, Haskell WL, Crow R, Sternfeld B, Oberman A, Armstrong MA, et al. Symptom-limited graded treadmill exercise testing in young adults in the CARDIA study. Med Sci Sports Exerc. 1992;24:177-83.

60. Sidney S, Sternfeld B, Haskell WL, Quesenberry CP Jr, Crow RS, Thomas RJ. Seven-year change in graded exercise treadmill test performance in young adults in the CARDIA study. Cardiovascular risk factors in young adults. Med Sci Sports Exerc. 1998;30:427-33.

61. Singhal N, Siddhu A. Association of leisure-time physical activity with cardiorespiratory fitness in Indian men. J Phys Act Health. 2014;11:296-302. https://doi.org/10.1123/jpah.2012-0029.

62. Strand LB, Laugsand LE, Wisloff U, Nes BM, Vatten L, Janszky I. Insomnia symptoms and cardiorespiratory fitness in healthy individuals: the NordTrondelag Health Study (HUNT). Sleep. 2013;36:99-108. https://doi.org/1 0.5665 /sleep. 2310

63. Sui $X$, Zhang J, Lee DC, Church TS, Lu W, Liu J, et al. Physical activity/fitness peaks during perimenopause and BMI change patterns are not associated with baseline activity/fitness in women: a longitudinal study with a median 7-year follow-up. Br J Sports Med. 2013;47:77-82. https://doi.org/10.1136/ bjsports-2011-090888.

64. Svedenkrans J, Henckel E, Kowalski J, Norman M, Bohlin K. Long-term impact of preterm birth on exercise capacity in healthy young men: a national population-based cohort study. PLoS ONE. 2013;8:e80869. https:// doi.org/10.1371/journal.pone.0080869

65. Talbot LA, Metter EJ, Fleg JL. Leisure-time physical activities and their relationship to cardiorespiratory fitness in healthy men and women 18-95 years old. Med Sci Sports Exerc. 2000;32:417-25.

66. Tammelin T, Nayha S, Rintamaki H. Cardiorespiratory fitness of males and females of northern Finland birth cohort of 1966 at age 31. Int J Sports Med. 2004;25:547-52. https://doi.org/10.1055/s-2004-820949.

67. Thai A, Papapanou PN, Jacobs DR Jr, Desvarieux M, Demmer RT. Periodontal infection and cardiorespiratory fitness in younger adults: results from continuous National Health and Nutrition Examination Survey 1999-2004. PLoS ONE. 2014;9. https://doi.org/10.1371/journal.pone.0092441.

68. Tobita Y, Kusaka Y, Ohtaki H, Hashizume K. Factors affecting the estimated maximal oxygen uptake: a follow-up study of participants in the total health promotion plan. Environ Health Prev Med. 2003;8:173-7.

69. Toth MJ, Gardner AW, Ades PA, Poehlman ET. Contribution of body composition and physical activity to age-related decline in peak VO2 in men and women. J Appl Physiol. 1994;77:647-52.

70. Twisk JWR, Kemper HCG, Van Mechelen W, Post GB. Which lifestyle parameters discriminate high-from low-risk participants for coronary heart disease risk factors. Longitudinal analysis covering adolescence and young adulthood. J Cardiovasc Risk. 1997;4:393-400.

71. Vrijlandt EJ, Gerritsen J, Boezen HM, Grevink RG, Duiverman EJ. Lung function and exercise capacity in young adults born prematurely. Am J Respir Crit Care Med. 2006;173:890-6.

72. Wong SYS, Chan FWK, Lee CK, Li M, Yeung F, Lum CCM, et al. Maximum oxygen uptake and body composition of healthy Hong Kong Chinese adult men and women aged 20-64 years. J Sports Sci. 2008;26:295-302. https:// doi.org/10.1080/02640410701552658.

73. Yu R, Yau F, Ho SC, Woo J. Longitudinal changes in physical activity levels over 5 years and relationship to cardiorespiratory fitness in Chinese midlife women. J Sports Med Phys Fitness. 2013;53:680-6.

74. Nagaya T, Kondo Y, Shibata T. Effects of sedentary work on physical fitness and serum cholesterol profile in middle-aged male workers. Int Arch Occup Environ Health. 2001;74:366-70.

75. Lopez-Martinez S, Sanchez-Lopez M, Solera-Martinez M, Arias-Palencia N, FuentesChacon RM, Martinez-Vizcaino V. Physical activity, fitness, and metabolic syndrome in young adults. Int J Sport Nutr Exerc Metab. 2013;23:312-21.

76. Lefevre J, Philippaerts RM, Delvaux K, Thomis M, Vanreusel B, Eynde BV, et al. Daily physical activity and physical fitness from adolescence to 
adulthood: a longitudinal study. Am J Hum Biol. 2000;12:487-97. https://doi. org/10.1002/1520-6300(200007/08)12:4<487:AID-AJHB8>3.0.CO;2-W.

77. Carnethon MR, Gulati M, Greenland P. Prevalence and cardiovascular disease correlates of low cardiorespiratory fitness in adolescents and adults. JAMA. 2005;294:2981-8. https://doi.org/10.1001/jama.294.23.2981.

78. Kim J-H, So W-Y. Correlation of waist circumference and body mass index with $\mathrm{VO}_{2}$ max in Korean adults. HealthMED. 2012;6:1632-5.

79. World Health Organization. Global action plan on physical activity 20182030: more active people for a healthier world. Geneva: WHO; 2018.

80. World Health Organization. Global action plan for the prevention and control of noncommunicable diseases 2013-2020. Geneva: WHO; 2013.

81. Warburton DER, Bredin SSD. Health benefits of physical activity: a systematic review of current systematic reviews. Curr Opin Cardiol. 2017;32:541-56. https://doi.org/10.1097/hco.0000000000000437.

82. World Health Organization. Global recommendations on physical activity for health. Appendix 5. Geneva: WHO; 2010

83. Hill A, Lupton H. Muscular exercise, lactic acid, and the supply and utilization of oxygen. QJM. 1923;0s-16(62):135-71.

84. Després JP. Physical activity, sedentary behaviours, and cardiovascular health: when will cardiorespiratory fitness become a vital sign? Can J Cardiol. 2016:32:505-13. https://doi.org/10.1016/j.cjca.2015.12.006.

85. Kodama S, Saito K, Tanaka S, et al. Cardiorespiratory fitness as a quantitative predictor of all-cause mortality and cardiovascular events in healthy men and women: a meta-analysis. JAMA. 2009;301:2024-35. https://doi.org/10.1 001/jama.2009.681

86. Lee DC, Artero EG, Sui X, Blair SN. Review: mortality trends in the general population: the importance of cardiorespiratory fitness. J Psychopharmacol. 2010;24(Suppl 4):27-35. https://doi.org/10.1177/1359786810382057.

87. Davidson T, Vainshelboim B, Kokkinos P, Myers J, Ross R. Cardiorespiratory fitness versus physical activity as predictors of all-cause mortality in men Am Heart J. 2018;196:156-62. https://doi.org/10.1016/j.ahj.2017.08.022.

88. Blair SN, Cheng Y, Holder JS. Is physical activity or physical fitness more important in defining health benefits? Med Sci Sports Exerc. 2001;33(Suppl 6):S379-99 discussion S419-20.

89. Blair SN, Kohl HW 3rd, Paffenbarger RS Jr, Clark DG, Cooper KH, Gibbons LW. Physical fitness and all-cause mortality: a prospective study of healthy men and women. JAMA. 1989;262:2395-401. https://doi.org/10.1001/jama.1989. 03430170057028.

90. Schmid D, Leitzmann MF. Cardiorespiratory fitness as predictor of cancer mortality: a systematic review and meta-analysis. Ann Oncol. 2015;26:272-8. https://doi.org/10.1093/annonc/mdu250.

91. Sui X, Laditka JN, Church TS, Hardin JW, Chase N, Davis K, et al. Prospective study of cardiorespiratory fitness and depressive symptoms in women and men. J Psychiatr Res. 2009;43:546-52. https://doi.org/10.1016/j.jpsychires.2 008.08.002.

92. Aberg MA, Waern M, Nyberg J, Pedersen NL, Bergh Y, Aberg ND, et al. Cardiovascular fitness in males at age 18 and risk of serious depression in adulthood: Swedish prospective population-based study. Br J Psychiatry. 2012; 201:352-9. https:/doi.org/10.1192/bjp.bp.111.103416.

93. Lee DC, Sui X, Church TS, Lavie CJ, Jackson AS, Blair SN. Changes in fitness and fatness on the development of cardiovascular disease risk factors: hypertension, metabolic syndrome, and hypercholesterolemia. J Am Coll Cardiol. 2012;59:665-72. https://doi.org/10.1016/j.jacc.2011.11.013.

94. LaMonte MJ, Barlow CE, Jurca R, Kampert JB, Church TS, Blair SN. Cardiorespiratory fitness is inversely associated with the incidence of metabolic syndrome: a prospective study of men and women. Circulation. 2005;112:505-12. https://doi.org/10.1161/circulationaha.104.503805.

95. Myers J, Prakash M, Froelicher V, Do D, Partington S, Atwood JE. Exercise capacity and mortality among men referred for exercise testing. N Engl J Med. 2002;346:793-801. https://doi.org/10.1056/ NEJMoa011858.

96. Bouchard C, An P, Rice T, Skinner JS, Wilmore JH, Gagnon J, et al. Familial aggregation of $\mathrm{VO}_{2 \max }$ response to exercise training: results from the HERITAGE family study. J Appl Physiol. 1999;87:1003-8. https://doi.org/1 0.1152/jappl.1999.87.3.1003

97. Bouchard C, Lesage R, Lortie G, Simoneau JA, Hamel P, Boulay MR, et al. Aerobic performance in brothers, dizygotic and monozygotic twins. Med Sci Sports Exerc. 1986;18:639-46.

98. Ortega FB, Ruiz JR, Castillo MJ, Sjöström M. Physical fitness in childhood and adolescence: a powerful marker of health. Int J Obes. 2007:32:1-11. https:// doi.org/10.1038/s.ijo.0803774.
99. Schneider J. Age dependency of oxygen uptake and related parameters in exercise testing: an expert opinion on reference values suitable for adults. Lung. 2013;191:449-58. https://doi.org/10.1007/s00408-013-9483-3.

100. Al-Mallah MH, Juraschek SP, Whelton S, Dardari ZA, Ehrman JK, Michos ED, et al. Sex differences in cardiorespiratory fitness and all-cause mortality: the Henry Ford Exerclse Testing (FIT) Project. Mayo Clin Proc. 2016;91:755-62. https://doi.org/10.1016/j.mayocp.2016.04.002.

101. Baumeister SE, Finger JD, Gläser S, Dörr M, Markus MR, Ewert R, et al. Alcohol consumption and cardiorespiratory fitness in five population-based studies. Eur J Prev Cardiol. 2018;25:164-72. https://doi.org/10.1177/20474 87317738594

102. Ombrellaro KJ, Perumal N, Zeiher J, Hoebel J, Ittermann T, Ewert R, et al. Socioeconomic correlates and determinants of cardiorespiratory fitness in the general adult population: a systematic review and meta-analysis. Sports Med Open. 2018;4:25. https://doi.org/10.1186/s40798-018-0137-0.

103. Hoehner CM, Allen P, Barlow CE, Marx CM, Brownson RC, Schootman M. Understanding the independent and joint associations of the home and workplace built environments on cardiorespiratory fitness and body mass index. Am J Epidemiol. 2013;178:1094-105. https://doi.org/10.1093/aje/kwt111.

104. Butland B, Jebb S, Kopelman P, McPherson K, Thomas S, Mardell J, et al. Foresight - tackling obesities: future choices: Government Office for Science; 2007.

105. Davison KK, Birch LL. Childhood overweight: a contextual model and recommendations for future research. Obes Rev. 2001;2:159-71.

106. Bauman AE, Reis RS, Sallis JF, Wells JC, Loos RJF, Martin BW. Correlates of physical activity: why are some people physically active and others not? Lancet. 2012;380:258-71. https://doi.org/10.1016/S0140-6736(12)60735-1.

107. Sallis JF, Cervero RB, Ascher W, Henderson KA, Kraft MK, Kerr J. An ecological approach to creating active living communities. Annu Rev Public Health. 2006;27:297-322. https://doi.org/10.1146/annurev. publhealth.27.021405.102100.

108. Perumal N, Mensink GBM, Keil T, Finger JD. Why are some people more fit than others? Correlates and determinants of cardiorespiratory fitness in adults: protocol for a systematic review. Syst Rev. 2017;6:102. https://doi. org/10.1186/s13643-017-0497-4.

109. Albouaini K, Egred M, Alahmar A. Cardiopulmonary exercise testing and its application. Postgrad Med J. 2007;83:675-82. https://doi.org/10.1136/hrt.2 007.121558.

110. Day JR, Rossiter HB, Coats EM, Skasick A, Whipp BJ. The maximally attainable $\dot{\mathrm{V}}_{2}$ during exercise in humans: the peak vs. maximum issue. J Appl Physiol. 2003:95:1901-7. https://doi.org/10.1152/japplphysiol.00024.2003.

111. Beltz NM, Gibson AL, Janot JM, Kravitz L, Mermier CM, Dalleck LC. Graded exercise testing protocols for the determination of $\mathrm{VO}_{2}$ max: historical perspectives, progress, and future considerations. J Sports Med. 2016;2016: 3968393. https://doi.org/10.1155/2016/3968393.

112. Victora CG, Huttly SR, Fuchs SC, Olinto MT. The role of conceptual frameworks in epidemiological analysis: a hierarchical approach. Int J Epidemiol. 1997;26:224-7. https:/doi.org/10.1093/ije/26.1.224.

113. Williams CJ, Williams MG, Eynon N, Ashton KJ, Little JP, Wisloff U, et al. Genes to predict $\mathrm{VO}_{2 \text { max }}$ trainability: a systematic review. BMC Genomics. 2017;18(Suppl 8):831. https://doi.org/10.1186/s12864-017-4192-6.

114. National Heart, Lung, and Blood Institute. Quality assessment tool for observational cohort and cross-sectional studies: Department of Health and Human Services; 2014.

115. Sallis JF, Prochaska JJ, Taylor WC. A review of correlates of physical activity of children and adolescents. Med Sci Sports Exerc. 2000;32:963-75.

116. Ferreira I, Horst KVD, Wendel-Vos W, Kremers S, Lenthe FJV, Brug J. Environmental correlates of physical activity in youth - a review and update. Obes Rev. 2007;8:129-54. https:/doi.org/10.1111/j.1467-789X.2006.00264.x.

117. Gorely T, Marshall SJ, Biddle SJH. Couch kids: correlates of television viewing among youth. Int J Behav Med. 2004;11:152-63. https://doi.org/10.1207/ s15327558ijbm1103_4.

118. Katzel LI, Sorkin JD, Fleg JL. A comparison of longitudinal changes in aerobic fitness in older endurance athletes and sedentary men. J Am Geriatr Soc. 2001:49:1657-64.

119. Ross R, Blair SN, Arena R, Church TS, Despres JP, Franklin BA, et al. Importance of assessing cardiorespiratory fitness in clinical practice: a case for fitness as a clinical vital sign: a scientific statement from the American Heart Association. Circulation. 2016;134:e653-e99. https://doi.org/10.1161/cir.0000000000000461.

120. Hawkins SA, Wiswell RA. Rate and mechanism of maximal oxygen consumption decline with aging. Sports Med. 2003;33:877-88. https://doi. org/10.2165/00007256-200333120-00002. 
121. Zoeller RF. Gender differences in cardiorespiratory fitness with advancing age: is the age-associated decline in VO2max more rapid in men and do older men and women respond differently to exercise? Am J Lifestyle Med. 2008:2:492-9. https://doi.org/10.1177/1559827608323631.

122. Shvartz E, Reibold RC. Aerobic fitness norms for males and females aged 6 to 75 years: a review. Aviat Space Environ Med. 1990;61:3-11.

123. Paap D, Takken T. Reference values for cardiopulmonary exercise testing in healthy adults: a systematic review. Expert Rev Cardiovasc Ther. 2014;12: 1439-53. https://doi.org/10.1586/14779072.2014.985657.

124. Wilson TM, Tanaka H. Meta-analysis of the age-associated decline in maximal aerobic capacity in men: relation to training status. Am J Physiol Heart Circ Physiol. 2000;278:H829-34. https://doi.org/10.1152/ ajpheart.2000.278.3.H829.

125. Fitzgerald MD, Tanaka H, Tran ZV, Seals DR. Age-related declines in maximal aerobic capacity in regularly exercising vs. sedentary women: a meta-analysis. J Appl Physiol. 1997;83:160-5. https://doi.org/10.1152/jappl.1997.83.1.160.

126. Wang CY, Haskell WL, Farrell SW, Lamonte MJ, Blair SN, Curtin LR, et al. Cardiorespiratory fitness levels among US adults 20-49 years of age: findings from the 1999-2004 National Health and Nutrition Examination Survey. Am J Epidemiol. 2010;171:426-35. https://doi.org/10.1093/aje/kwp412.

127. Hakola L. Cardiorespiratory fitness and physical activity in older adults: a population-based study in men and women. Tampere: Kuopio Research Institute of Exercise Medicine; 2015

128. Loe H, Rognmo $\varnothing$, Saltin B, Wisløff U. Aerobic capacity reference data in 3816 healthy men and women 20-90 years. PLOS ONE. 2013;8:e64319. https://doi.org/10.1371/journal.pone.0064319.

129. O’Donoghue G, Kennedy A, Puggina A, Aleksovska K, Buck C, Burns C, et al. Socio-economic determinants of physical activity across the life course: a "DEterminants of Dlet and Physical Activity" (DEDIPAC) umbrella literature review. PLoS ONE. 2018;13:e0190737. https://doi.org/1 0.1371/journal.pone.0190737.

130. Giskes K, Avendano M, Brug J, Kunst AE. A systematic review of studies on socioeconomic inequalities in dietary intakes associated with weight gain and overweight/obesity conducted among European adults. Obes Rev. 2010;11:413-29. https://doi.org/10.1111/j.1467-789X.2009.00658.x.

131. Hiscock R, Bauld L, Amos A, Fidler JA, Munafò M. Socioeconomic status and smoking: a review. Ann N Y Acad Sci. 2012;1248:107-23. https://doi.org/1 0.1111/j.1749-6632.2011.06202.x.

132. Wang Y, Beydoun MA. The obesity epidemic in the United States-gender, age, socioeconomic, racial/ethnic, and geographic characteristics: a systematic review and meta-regression analysis. Epidemiol Rev. 2007;29:628. https://doi.org/10.1093/epirev/mxm007.

133. Omran AR. The epidemiologic transition: a theory of the epidemiology of population change. Milbank Q. 2005;83:731-57.

134. Dinsa GD, Goryakin Y, Fumagalli E, Suhrcke M. Obesity and socioeconomic status in developing countries: a systematic review. Obes Rev. 2012;13: 1067-79. https://doi.org/10.1111/j.1467-789X.2012.01017.x.

135. Fogelholm M. Physical activity, fitness and fatness: relations to mortality, morbidity and disease risk factors. A systematic review. Obes Rev. 2010;11: 202-21. https://doi.org/10.1111/j.1467-789X.2009.00653.x.

136. Ross R, Katzmarzyk PT. Cardiorespiratory fitness is associated with diminished total and abdominal obesity independent of body mass index. Int J Obes. 2003;27:204-10. https://doi.org/10.1038/sj.ijo.802222.

137. Hollmann W, Strüder HK. Sportmedizin. Grundlagen für körperliche Aktivität, Training und Präventivmedizin. [Sports medicine: fundamentals of physical activity, exercise and preventive medicine] 5th ed. Stuttgart and New York: Schattauer; 2009.

138. Benck LR, Cuttica MJ, Colangelo LA, Sidney S, Dransfield MT, Mannino DM, et al. Association between cardiorespiratory fitness and lung health from young adulthood to middle age. Am J Respir Crit Care Med. 2017;195:123643. https://doi.org/10.1164/rccm.201610-20890C.

139. Kaczynski AT, Manske SR, Mannell RC, Grewal K. Smoking and physical activity: a systematic review. Am J Health Behav. 2008;32:93-110. https://doi. org/10.5555/ajhb.2008.32.1.93.

140. Grønbæk M. Confounders of the relation between type of alcohol and cardiovascular disease. Ann Epidemiol. 2007;17:S13-5. https://doi.org/10.1 016/j.annepidem.2007.01.004.

141. Heidemann C, Scheidt-Nave C, Richter A, Mensink GBM. Dietary patterns are associated with cardiometabolic risk factors in a representative study population of German adults. Br J Nutr. 2011;106:1253-62. https://doi.org/1 $0.1017 /$ S0007114511001504.
142. Lahoz-Garcia N, Garcia-Hermoso A, Milla-Tobarra M, Diez-Fernandez A Soriano-Cano A, Martinez-Vizcaino V. Cardiorespiratory fitness as a mediator of the influence of diet on obesity in children. Nutrients. 2018;10:358. https://doi.org/10.3390/nu10030358.

143. Myers J, McAuley P, Lavie CJ, Despres J-P, Arena R, Kokkinos P. Physical activity and cardiorespiratory fitness as major markers of cardiovascular risk: their independent and interwoven importance to health status. Prog Cardiovasc Dis. 2015;57:306-14. https://doi.org/10.1016/j.pcad.2014.09.011.

144. Bouchard C, Blair SN, Katzmarzyk PT. Less sitting, more physical activity, or higher fitness? Mayo Clin Proc. 2015;90:1533-40. https://doi.org/10.1016/j. mayocp.2015.08.005.

145. Holtermann A, Hansen JV, Burr H, Søgaard K, Sjøgaard G. The health paradox of occupational and leisure-time physical activity. Br J Sports Med. 2012;46:291-5. https://doi.org/10.1136/bjsm.2010.079582.

146. Sisson SB, Camhi SM, Church TS, Martin CK, Tudor-Locke C, Bouchard C, et al. Leisure time sedentary behavior, occupational/domestic physical activity, and metabolic syndrome in U.S. men and women. Metab Syndr Relat Disord. 2009;7:529-36. https://doi.org/10.1089/met.2009.0023.

147. Pollock ML, Foster C, Schmidt D, Hellman C, Linnerud AC, Ward A. Comparative analysis of physiologic responses to three different maximal graded exercise test protocols in healthy women. Am Heart J. 1982;103: 363-73. https://doi.org/10.1016/0002-8703(82)90275-7.

148. Pollock ML, Bohannon RL, Cooper KH, Ayres JJ, Ward A, White SR, et al. A comparative analysis of four protocols for maximal treadmill stress testing. Am Heart J. 1976;92:39-46. https://doi.org/10.1016/S0002-8703(76)80401-2.

149. Evans HJL, Ferrar KE, Smith AE, Parfitt G, Eston RG. A systematic review of methods to predict maximal oxygen uptake from submaximal, open circuit spirometry in healthy adults. J Sci Med Sport. 2015;18:183-8. https://doi. org/10.1016/j.jsams.2014.03.006.

150. Hanley JA. Appropriate uses of multivariate analysis. Annu Rev Public Health. 1983:4:155-80.

151. Bauman AE, Sallis JF, Dzewaltowski DA, Owen N. Toward a better understanding of the influences on physical activity: the role of determinants, correlates, causal variables, mediators, moderators, and confounders. Am J Prev Med. 2002;23(Suppl 2):5-14. https://doi.org/10.1016/ S0749-3797(02)00469-5.

152. Bull FC, Maslin TS, Armstrong T. Global Physical Activity Questionnaire (GPAQ): nine country reliability and validity study. J Phys Act Health. 2009;6:790-804.

153. Finger JD, Tafforeau J, Gisle L, Oja L, Ziese T, Thelen J, et al. Development of the European Health Interview Survey - Physical Activity Questionnaire (EHIS-PAQ) to monitor physical activity in the European Union. Arch Public Health. 2015;73:59. https://doi.org/10.1186/s13690-015-0110-z.

154. Rezende LFM, Rodrigues Lopes M, Rey-López JP, Matsudo VKR, Luiz ODC. Sedentary behavior and health outcomes: an overview of systematic reviews. PLoS ONE. 2014;9:e105620. https://doi.org/10.1371/journal.pone.0105620.

155. European Commission. Policies and interventions to improve the nutritional intake and physical activity levels of Europeans: review of scientific evidence and policies on nutrition and physical activity. Brussels: EC; 2018. https://doi. org/10.2875/670056.

156. Meader N, King K, Wright K, Graham HM, Petticrew M, Power C, et al. Multiple risk behavior interventions: meta-analyses of RCTs. Am J Prev Med. 2017:53:e19-30. https://doi.org/10.1016/j.amepre.2017.01.032.

157. Laukkanen JA, Zaccardi F, Khan H, Kurl S, Jae SY, Rauramaa R. Long-term Change in Cardiorespiratory Fitness and All-Cause Mortality: A PopulationBased Follow-up Study. Mayo Clin Proc. 2016;91(9):1183-8.

158. Chen L, Kuang J, Pei JH, Chen HM, Chen Z, Li ZW, et al. Predictors of cardiorespiratory fitness in female and male adults with different body mass index: National Health and Nutrition Examination Survey 1999-2004 dataset. Ann Med. 2017;49(1):83-92. https://doi.org/10.1080/07853890.2016.1252056.

159. Jackowski SA, Eisenmann JC, Sherar LB, Bailey DA, Baxter-Jones ADG. Adolescent trajectories of aerobic fitness and adiposity as markers of cardiometabolic risk in adulthood. J Obes. 2017;2017:6471938. https://doi. org/10.1155/2017/6471938

160. Knaeps S, Bourgois JG, Charlier R, Mertens E, Lefevre J, Wijndaele K. Ten-year change in sedentary behaviour, moderate-to-vigorous physical activity, cardiorespiratory fitness and cardiometabolic risk: independent associations and mediation analysis. Br J Sports Med. 2018;52(16):1063-8. https://doi. org/10.1136/bjsports-2016-096083.

161. Shouval R, Katz S, Nagler A, Merkel D, Ben-Zvi I, Segev S, et al. Gender disparities in the functional significance of anemia among apparently healthy adults. Eur J Haematol. 2017;98(5):435-42. https://doi.org/10.1111/ejh.12849. 
162. Tripette J, Murakami H, Hara H, Kawakami R, Gando Y, Ohno H, et al. Caffeine consumption is associated with higher level of physical activity in Japanese women. Int J Sport Nutr Exerc Metab. 2018;28(5):474-9. https:// doi.org/10.1123/ijsnem.2017-0428.

163. Tsou PL, Wu CJ. Sex-dimorphic association of plasma fatty acids with cardiovascular fitness in young and middle-aged general adults: subsamples from NHANES 2003(-)2004. Nutrients. 2018;10(10). https://doi.org/10.3390/ nu10101558.

164. van der Velde J, Koster A, van der Berg J, Sep S, van der Kallen C, Dagnelie $P$, et al. Sedentary Behavior, Physical Activity, and Fitness-The Maastricht Study. Med Sci Sports Exerc. 2017;49(8):1583-91. https://doi.org/10.1249/ mss.0000000000001262.

165. Lorenzo S, Babb TG. Quantification of cardiorespiratory fitness in healthy nonobese and obese men and women. Chest. 2012;141(4):1031-9. https:// doi.org/10.1378/chest.11-1147.

\section{Publisher's Note}

Springer Nature remains neutral with regard to jurisdictional claims in published maps and institutional affiliations.

\section{Submit your manuscript to a SpringerOpen ${ }^{\odot}$ journal and benefit from:}

- Convenient online submission

- Rigorous peer review

- Open access: articles freely available online

High visibility within the field

- Retaining the copyright to your article

Submit your next manuscript at $\boldsymbol{\nabla}$ springeropen.com 